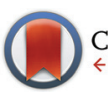

CrossMark $\leftarrow$ click for updates

Cite this: Dalton Trans., 2016, 45 7900

Received 29th February 2016, Accepted 25th March 2016

DOI: $10.1039 / c 6 d t 00808 a$

www.rsc.org/dalton

\section{Complexes of Group 2 dications with soft thioether- and selenoether-containing macrocycles $\dagger$}

\begin{abstract}
William Levason, David Pugh, Jamie M. Purkis and Gillian Reid*
A new route to cationic complexes of $\mathrm{Mg}, \mathrm{Ca}, \mathrm{Sr}$ and $\mathrm{Ba}$ with 18-membered ring $\mathrm{O}_{4} \mathrm{~S}_{2}, \mathrm{O}_{4} \mathrm{Se}_{2}$ and $\mathrm{O}_{2} \mathrm{~S}_{4}$ donor macrocycles from metal acetonitrile complexes with weakly coordinating $\left[\mathrm{BAr}^{\mathrm{F}}\right]^{-}$anions is described. The precursors used were $\left[\mathrm{M}(\mathrm{MeCN})_{x}\right][\mathrm{BAr}]_{2}(M=M g, x=6 ; M=C a, x=8)$ and $\left[\mathrm{M}^{\prime}(\mathrm{acacH})(\mathrm{MeCN})_{5}\right][\mathrm{BAr}]_{2}\left(\mathrm{M}^{\prime}=\mathrm{Sr}\right.$ or Ba). Reaction of these with the heterocrowns, [18]aneO $\mathrm{S}_{2}(1,4,10,13-$ tetraoxa-7,16-dithiacyclooctadecane), [18]ane ${ }_{4} \mathrm{Se}_{2}$ (1,4,10,13-tetraoxa-7,16-diselenacyclooctadecane) or [18]ane $\mathrm{O}_{2} \mathrm{~S}_{4}$ (1,10-dioxa-4,7,13,16-tetrathiacyclooctadecane) in anhydrous $\mathrm{CH}_{2} \mathrm{Cl}_{2}$ solution gave $\left[\mathrm{M}\right.$ (heterocrown) $\left.(\mathrm{MeCN})_{2}\right]\left[\mathrm{BAr}^{\mathrm{F}}\right]_{2}$ for $\mathrm{M}=\mathrm{Mg}$, Ca or Sr, whilst the larger Ba forms [Ba(heterocrown)(acacH)$(\mathrm{MeCN})][\mathrm{BAr}]_{2}$. The complexes have been characterised by microanalysis, $I \mathrm{R},{ }^{1} \mathrm{H}$ and ${ }^{13} \mathrm{C}\left\{{ }^{1} \mathrm{H}\right\} \mathrm{NMR}$ spectroscopy. X-ray crystal structures are reported for $\left[\mathrm{Ca}\left([18] \mathrm{aneO}_{2} \mathrm{~S}_{4}\right)\left(\mathrm{MeCN}_{2}\right][\mathrm{BAr}]_{2}\right.$, [Ca([18]aneO $\left.\mathrm{Se}_{2}\right)$ $\left.(\mathrm{MeCN})_{2}\right]\left[\mathrm{BAr}^{\mathrm{F}}\right]_{2}, \quad\left[\mathrm{Sr}\left([18] \mathrm{aneO}_{4} \mathrm{~S}_{2}\right)(\mathrm{MeCN})_{2}\right]\left[\mathrm{BAr}^{\mathrm{F}}\right]_{2}$, and $\left[\mathrm{Sr}\left([18] \mathrm{aneO}_{4} \mathrm{Se}_{2}\right)(\mathrm{MeCN})_{2}\right]\left[\mathrm{BAr}^{\mathrm{F}}\right]_{2}$ which contain 8 -coordinate metal centres with trans-nitrile ligands and $\kappa^{6}$-heterocrowns, and for the 9 -coordinate $\left[\mathrm{Ba}\left([18] \mathrm{aneO}_{4} \mathrm{Se}_{2}\right)(\mathrm{acacH})(\mathrm{MeCN})\right]\left[\mathrm{BAr}^{\mathrm{F}}\right]_{2}$. Adventitious hydrolysis of the magnesium complexes in solution results in six-coordinate complexes, $\left[\mathrm{Mg}\left(\mathrm{k}^{3}-[18] \mathrm{aneO}_{4} \mathrm{Se}_{2}\right)\left(\mathrm{OH}_{2}\right)_{2}(\mathrm{MeCN})\right]\left[\mathrm{BAr}^{\mathrm{F}}\right]_{2}$ and $\left[\mathrm{Mg}\left(\mathrm{k}^{3}-[18] \mathrm{aneO}_{4} \mathrm{~S}_{2}\right)-\right.$ $\left.\left(\mathrm{OH}_{2}\right)_{2}(\mathrm{MeCN})\right]\left[\mathrm{BAr}^{\mathrm{F}}\right]_{2}$, whose structures were determined. X-ray crystal structures are also reported for $\left[\mathrm{Mg}(\mathrm{MeCN})_{6}\right][\mathrm{BAr}]_{2},\left[\mathrm{M}(\mathrm{MeCN})_{8}\right]\left[\mathrm{BAr}{ }^{\mathrm{F}}\right]_{2}(\mathrm{M}=\mathrm{Ca}, \mathrm{Sr})$ and $\left[\mathrm{Ca}(18-\mathrm{crown}-6)(\mathrm{MeCN})_{2}\right]\left[\mathrm{BAr}{ }^{\mathrm{F}}\right]_{2}$.
\end{abstract}

\section{Introduction}

The chemistries of the heavier alkaline earth metals $(\mathrm{Ca}, \mathrm{Sr}$ and $\mathrm{Ba}$ ) are dominated by the hard $\mathrm{M}^{2+}$ cations and their salts with oxo-anions or halides. ${ }^{1}$ Barium and strontium salts have niche applications, for example $\mathrm{BaSO}_{4}$ in the oil industry and medicine, but the key importance is of calcium salts, which range from engineering and construction $\left(\mathrm{CaCO}_{3}, \mathrm{CaSO}_{4}\right.$, $\mathrm{Ca}(\mathrm{OH})_{2}$ ) to apatite in mammalian bones and $\mathrm{CaCO}_{3}$ in corals and shells. For many years their coordination chemistry with neutral ligands was very limited and the organometallic chemistry among the most restricted in the main group. ${ }^{1-3}$ However, the last 20 years have seen the development of the coordination chemistry with both charged and neutral oxygen and nitrogen ligands, ${ }^{4}$ and despite the synthetic challenges and high reactivities, of a significant organometallic chemistry. ${ }^{5}$ The coordination chemistry of magnesium is more familiar, not least because of the importance of chlorophyll and chlorophyll model compounds, ${ }^{4}$ while Grignard reagents have been

School of Chemistry, University of Southampton, Highfield, Southampton SO17 1BJ, UK.E-mail: g.reid@soton.ac.uk

$\dagger$ Electronic supplementary information (ESI) available: X-ray data on [Sr $\left.(\mathrm{MeCN})_{8}\right]\left[\mathrm{BAr}{ }^{\mathrm{F}}\right]_{2}$ and $\left[\mathrm{Ca}\left(18-\right.\right.$ crown-6) $\left.(\mathrm{MeCN})_{2}\right]\left[\mathrm{BAr}{ }^{\mathrm{F}}\right]_{2}$. Spectroscopic data on the complexes. CCDC 1455287-1455298. For ESI and crystallographic data in CIF or other electronic format see DOI: 10.1039/c6dt00808a known for over 100 years. ${ }^{6}$ Despite these recent developments, the synthesis of complexes of these elements retains significant challenges, not least the lability of the metal centres, which often results in unpredicted products or mixtures, even in apparently simple systems. ${ }^{7}$ We have recently been exploring the use of weakly coordinating fluorinated tetraarylborate anions, such as $\left[\mathrm{B}\left\{3,5-\left(\mathrm{CF}_{3}\right)_{2} \mathrm{C}_{6} \mathrm{H}_{3}\right\}_{4}\right]^{-}$(hereafter $\left.\mathrm{BAr}^{\mathrm{F}}\right)$ to obtain unusual complexes of the Group 1 metal ions, including the synthesis of homoleptic neutral diphosphine complexes of lithium and sodium, $\left[\mathrm{M}(\mathrm{L}-\mathrm{L})_{3}\right]\left[\mathrm{BAr}^{\mathrm{F}}\right](\mathrm{M}=\mathrm{Li}$ or $\mathrm{Na}$; $\mathrm{L}-\mathrm{L}=$ $\mathrm{Me}_{2} \mathrm{PCH}_{2} \mathrm{CH}_{2} \mathrm{PMe}_{2}$ or $\left.O-\mathrm{C}_{6} \mathrm{H}_{4}\left(\mathrm{PMe}_{2}\right)_{2}\right),{ }^{8}$ the homoleptic octathioether complex of sodium, $\left[\mathrm{Na}\left([24] \mathrm{aneS}_{8}\right)\right]\left[\mathrm{BAr}^{\mathrm{F}}\right]$ ([24] aneS $_{8}=1,4,7,10,13,16,19,22$-octathiacyclotetracosane), ${ }^{9}$ complexes of Li-Cs of $\mathrm{N}_{3^{-}}, \mathrm{N}_{4^{-}}$and $\mathrm{N}_{6}$-donor azamacrocycles, ${ }^{10}$ and with the heterocrowns, [18] aneO $_{2} \mathrm{~S}_{4}$ (1,10-dioxa-4,7,13,16-tetrathiacyclooctadecane), [18] $\mathrm{aneO}_{4} \mathrm{~S}_{2} \quad(1,4,10,13$-tetraoxa-7,16dithiacyclooctadecane) and [18] $\mathrm{aneO}_{4} \mathrm{Se}_{2}$ (1,4,10,13-tetraoxa7,16-diselenacyclooctadecane). ${ }^{11}$ These contain rare coordination of the neutral sulfur or selenium donors to the alkali metal centre. Heterocrowns containing S- or Se-donor atoms have an extensive chemistry with $\mathrm{d}$ - and p-block metals, ${ }^{12}$ but examples with the s-block elements are few. In addition to the Group 1 examples described above, we reported examples of neutral eight-coordinate complexes of $\mathrm{CaI}_{2}$ and $\mathrm{SrI}_{2}$ with [18] aneO $_{4} \mathrm{~S}_{2}$, [18] $\mathrm{aneO}_{2} \mathrm{~S}_{4}$ and [18] $\mathrm{aneO}_{4} \mathrm{Se}_{2} .{ }^{13}$ The complex $\left[\mathrm{Ca}\left(\mathrm{ClO}_{4}\right)_{2}\left([18] \mathrm{aneO}_{4} \mathrm{~S}_{2}\right)\right],{ }^{14}$ the lamellar polymer $\left[\mathrm{Ba}\left\{\mathrm{Cu}(\mathrm{SCN})_{3}([18]-\right.\right.$ 
aneO $\left.\left.\left._{4} \mathrm{~S}_{2}\right)\right\}\right],{ }^{15}$ and $\left[\mathrm{Mg}\left(\mathrm{R} \text {-benzo[18] } \mathrm{aneO}_{4} \mathrm{~S}_{2}\right)\right]^{16}$ (R-benzo[18]aneO $_{4} \mathrm{~S}_{2}=\mathrm{R}$-benzo-substituted [18] $\mathrm{aneO}_{4} \mathrm{~S}_{2}$ ) are also known. The present paper describes the synthesis, structures and spectroscopic properties of complexes of [18]aneO $\mathrm{S}_{4} \mathrm{~S}_{2}$, [18]aneO ${ }_{2} \mathrm{~S}_{4}$ and [18] $\mathrm{aneO}_{4} \mathrm{Se}_{2}$ with $\mathrm{Mg}, \mathrm{Ca}, \mathrm{Sr}$ and $\mathrm{Ba}$ dications, utilising the $\left[\mathrm{BAr}^{\mathrm{F}}\right]^{-}$anions to afford soluble cationic precursor species.

\section{Experimental}

All preparations were carried out under a dry dinitrogen atmosphere using standard Schlenk and glove box techniques. Anhydrous $\mathrm{MgI}_{2}, \mathrm{CaI}_{2}, \mathrm{SrI}_{2}$ and $\mathrm{BaI}_{2}$ were purchased from Sigma and used as received. Anhydrous $\left[\operatorname{Sr}(\mathrm{acac})_{2}\right]$ was prepared by dehydrating commercial $\left[\mathrm{Sr}(\mathrm{acac})_{2}\left(\mathrm{H}_{2} \mathrm{O}\right)_{2}\right]$ (Sigma): the hydrate was suspended in $\mathrm{CH}_{2} \mathrm{Cl}_{2}$ over $4 \AA$ molecular sieves and stirred gently for 2 weeks, then the suspension was decanted away and concentrated to dryness. $\left[\mathrm{Ba}(\mathrm{acac})_{2}\right]$ was synthesized by reacting $\mathrm{BaI}_{2}$ and $\mathrm{K}(\mathrm{acac})$ in a $1: 2$ molar ratio in MeCN followed by extraction of KI with anhydrous acetone. $\mathrm{Na}\left[\mathrm{BAr}{ }^{\mathrm{F}}\right]$ and $\left[\mathrm{H}\left(\mathrm{OEt}_{2}\right)_{2}\right]\left[\mathrm{BAr}^{\mathrm{F}}\right]$ were synthesized using Brookhart's procedure. ${ }^{17}$ 18-Crown- 6 was purchased from Sigma and dried using $\mathrm{SOCl}_{2}$ and the macrocycles $[18] \mathrm{aneO}_{4} \mathrm{~S}_{2}$, [18] $\mathrm{aneO}_{2} \mathrm{~S}_{4}$ and [18] $\mathrm{aneO}_{4} \mathrm{Se}_{2}$ were synthesized using literature procedures. ${ }^{18}$ $\mathrm{CH}_{2} \mathrm{Cl}_{2}$ and $\mathrm{MeCN}$ were dried by distillation from $\mathrm{CaH}_{2}$ and $n$-hexane distilled from $\mathrm{Na} / \mathrm{K}$ alloy. ${ }^{1} \mathrm{H}$ and ${ }^{13} \mathrm{C}\left\{{ }^{1} \mathrm{H}\right\} \mathrm{NMR}$ spectra were recorded in $\mathrm{CD}_{2} \mathrm{Cl}_{2}$ solution at $298 \mathrm{~K}$ using a Bruker AV II- 400 spectrometer and are referenced to the residual $\mathrm{CH}_{2} \mathrm{Cl}_{2}$ resonance. IR spectra were recorded as mulls between CsI plates using a Perkin-Elmer Spectrum 100 spectrometer over the range $4000-200 \mathrm{~cm}^{-1}$. Microanalyses were undertaken at London Metropolitan University.

\section{X-ray crystallography}

Crystals were obtained as described below. Details of the crystallographic data collection and refinement are in Table 1. Diffractometer: Rigaku AFC12 goniometer equipped with an enhanced sensitivity (HG) Saturn $724+$ detector mounted at the window of an FR-E+ SuperBright molybdenum rotating anode generator $\left(\lambda_{1}=0.71073 \AA\right)$ with VHF Varimax optics $(70$ or $110 \mu \mathrm{m}$ focus). Cell determination and data collection: CrystalClear-SM Expert $3.1 \mathrm{~b} 27$, data reduction, cell refinement, and absorption correction: CrystalClear-SM Expert 2.1. ${ }^{19}$ Structure solution and refinement were carried out using WinGX and software packages within. ${ }^{20}$ Disorder in the $\mathrm{CF}_{3}$ groups of the $\left[\mathrm{BAr}^{\mathrm{F}}\right]^{-}$anions was present in all of the structures, which is often observed in compounds containing $\left[\mathrm{BAr}^{\mathrm{F}}\right]^{-},{ }^{10,11}$ and this was satisfactorily modelled using DFIX, DANG, ISOR, DELU, and SIMU restraints. Positional disorder was also present in the macrocycle ligands and was modelled similarly. The dataset for $\left[\mathrm{Ca}\left([18] \mathrm{aneO}_{4} \mathrm{~S}_{2}\right)(\right.$ bipy $\left.)\right]\left[\mathrm{BAr}{ }^{\mathrm{F}}\right]_{2}$ was collected from a poor-quality crystal which was very weakly diffracting at high angles, thus leading to very high $R_{\text {int }}$ values. Repeated attempts at recrystallization of the sample failed to afford better quality crystals. A large amount of residual electron density was located $\sim 1.05 \AA$ from an Se atom in $\left[\mathrm{Ba}\left([18] \mathrm{aneO}_{4} \mathrm{Se}_{2}\right)(\mathrm{acacH})\right.$ -
$(\mathrm{MeCN})]\left[\mathrm{BAr}^{\mathrm{F}}\right]_{2}$ which could not be satisfactorily modelled as either disordered Se or disordered carbon. $\mathrm{H}$-atoms were placed in geometrically-assigned positions with $\mathrm{C}-\mathrm{H}$ distances of $0.95 \AA(\mathrm{CH}), 0.98 \AA\left(\mathrm{CH}_{3}\right)$ or $0.99 \AA\left(\mathrm{CH}_{2}\right)$ and refined using a riding model with $U_{\text {iso }}(\mathrm{H})=1.2 U_{\text {eq }}(\mathrm{C})\left(\mathrm{CH}, \mathrm{CH}_{2}\right)$ or $1.5 U_{\mathrm{eq}}(\mathrm{C})$ $\left(\mathrm{CH}_{3}\right)$. enCIFer was used to prepare material for publication. ${ }^{21}$ CCDC reference numbers $1455287 \quad\left[\mathrm{Mg}(\mathrm{MeCN})_{6}\right]\left[\mathrm{BAr}^{\mathrm{F}}\right]_{2}$, $1455288\left[\mathrm{Mg}\left(\mathrm{K}^{3}-[18] \mathrm{aneO}_{4} \mathrm{~S}_{2}\right)\left(\mathrm{OH}_{2}\right)_{2}(\mathrm{MeCN})\right]\left[\mathrm{BAr}^{\mathrm{F}}\right]_{2}, \quad 1455289$ $\left[\mathrm{Mg}\left(\kappa^{3}-[18] \mathrm{aneO}_{4} \mathrm{Se}_{2}\right)\left(\mathrm{OH}_{2}\right)_{2}\left(\mathrm{MeCN}^{2}\right]\left[\mathrm{BAr}^{\mathrm{F}}\right]_{2}, \quad 1455290\right.$ $\left[\mathrm{Ca}(\mathrm{MeCN})_{8}\right]\left[\mathrm{BAr}^{\mathrm{F}}\right]_{2}, 1455291\left[\mathrm{Ca}(18 \text {-crown-6)(MeCN })_{2}\right]\left[\mathrm{BAr}^{\mathrm{F}}\right]_{2}$, $1455292\left[\mathrm{Ca}\left([18] \mathrm{aneO}_{2} \mathrm{~S}_{4}\right)(\mathrm{MeCN})_{2}\right]\left[\mathrm{BAr}^{\mathrm{F}}\right]_{2}, \quad 1455293$ $\left[\mathrm{Ca}\left([18] \mathrm{aneO}_{4} \mathrm{Se}_{2}\right)\left(\mathrm{MeCN}_{2}\right]\left[\mathrm{BAr}^{\mathrm{F}}\right]_{2}, 1455294\left[\mathrm{Ca}\left([18] \mathrm{aneO}_{4} \mathrm{~S}_{2}\right)-\right.\right.$ (bipy) $]\left[\mathrm{BAr}^{\mathrm{F}}\right]_{2}, \quad 1455295 \quad\left[\mathrm{Sr}(\mathrm{MeCN})_{8}\right]\left[\mathrm{BAr}^{\mathrm{F}}\right]_{2}, \quad 1455296$ $\left[\mathrm{Sr}\left([18] \mathrm{aneO}_{4} \mathrm{~S}_{2}\right)\left(\mathrm{MeCN}_{2}\right]\left[\mathrm{BAr}^{\mathrm{F}}\right]_{2}, \quad 1455297 \quad\left[\mathrm{Sr}\left([18] \mathrm{aneO}_{4} \mathrm{Se}_{2}\right)-\right.\right.$ $\left.(\mathrm{MeCN})_{2}\right]\left[\mathrm{BAr}^{\mathrm{F}}\right]_{2}$ and $1455298 \quad\left[\mathrm{Ba}\left([18] \mathrm{aneO}_{4} \mathrm{Se}_{2}\right)(\mathrm{acacH})-\right.$ $(\mathrm{MeCN})]\left[\mathrm{BAr}^{\mathrm{F}}\right]_{2}$ contain crystallographic data in CIF format.

$\left[\mathrm{Mg}(\mathrm{MeCN})_{6}\right]\left[\mathrm{BAr}^{\mathrm{F}}\right]_{2}$. A mixture of $\mathrm{MgI}_{2}(0.34 \mathrm{~g}, 1.21 \mathrm{mmol})$ and $\mathrm{Na}\left[\mathrm{BAr}^{\mathrm{F}}\right](2.50 \mathrm{~g}, 2.43 \mathrm{mmol})$ were dissolved in MeCN $(10 \mathrm{~mL})$ and stirred for $24 \mathrm{~h}$. Solvents were removed, the residue extracted with $\mathrm{Et}_{2} \mathrm{O}(20 \mathrm{~mL})$, filtered and dried in vacuo. The solid was redissolved in MeCN $(5 \mathrm{~mL})$, stirred for $30 \mathrm{~min}$ and then taken to dryness again. The resulting solid was dissolved in $\mathrm{CH}_{2} \mathrm{Cl}_{2}(10 \mathrm{~mL})$ and $n$-hexane $(100 \mathrm{~mL})$ was added to precipitate an off-white solid. Yield: $0.55 \mathrm{~g}, 23 \%$. Crystals were grown by vapour diffusion of $n$-hexane into a concentrated solution in $\mathrm{CH}_{2} \mathrm{Cl}_{2}$. Anal. Required for $\mathrm{C}_{76} \mathrm{H}_{42} \mathrm{~B}_{2} \mathrm{~F}_{48} \mathrm{MgN}_{6}$ (1997.09): C, 45.71; H, 2.12; N, 4.21. Found: C, $45.61 ; \mathrm{H}, 2.02 ; \mathrm{N}, 4.17 \% .{ }^{1} \mathrm{H}$ NMR $\left(\mathrm{CD}_{2} \mathrm{Cl}_{2}, 298 \mathrm{~K}\right): \delta=7.74$ (s, [16H], BAr $\left.{ }^{\mathrm{F}} \mathrm{H} 2 / 6\right), 7.60$ (s, [8H], BAr $\left.{ }^{\mathrm{F}} \mathrm{H} 4\right), 2.16$ (s, [18H], $\mathrm{CH}_{3}$, MeCN). ${ }^{13} \mathrm{C}\left\{{ }^{1} \mathrm{H}\right\}$ NMR $\left(\mathrm{CD}_{2} \mathrm{Cl}_{2}, 298 \mathrm{~K}\right): \delta=162.30$ $\left(\mathrm{q},{ }^{1} J_{\mathrm{C}-\mathrm{B}}=50.0 \mathrm{~Hz}, \mathrm{BAr}^{\mathrm{F}} \mathrm{C} 1\right), 134.92\left(\mathrm{BAr}^{\mathrm{F}} \mathrm{C} 2 / 6\right), 129.30(\mathrm{q}$, $\left.{ }^{2} J_{\mathrm{C}-\mathrm{F}}=32 \mathrm{~Hz}, \mathrm{BAr}^{\mathrm{F}} \mathrm{C} 3 / 5\right), 124.73\left(\mathrm{q},{ }^{1} J_{\mathrm{C}-\mathrm{F}}=272 \mathrm{~Hz}, \mathrm{CF}_{3}\right)$, 118.16 (BAr $\left.{ }^{\mathrm{F}}, \mathrm{C} 4\right), 117.87$ (C, MeCN), $2.18\left(\mathrm{CH}_{3}, \mathrm{MeCN}\right)$. IR (Nujol/cm ${ }^{-1}$ ): 2321, 2295 (MeCN), 1611, 1357, 1282, 1261, 1111, 1022, 883, 801, 669, 403.

$\left[\mathrm{Ca}(\mathrm{MeCN})_{8}\right]\left[\mathrm{BAr}^{\mathrm{F}}\right]_{2}$. A mixture of $\mathrm{CaI}_{2}(0.25 \mathrm{~g}, 0.85 \mathrm{mmol})$ and $\mathrm{Na}\left[\mathrm{BAr}{ }^{\mathrm{F}}\right](1.50 \mathrm{~g}, 1.69 \mathrm{mmol})$ were dissolved in MeCN $(10 \mathrm{~mL})$ and stirred for $24 \mathrm{~h}$, affording a yellow solution. Solvents were removed, the residue extracted with $\mathrm{Et}_{2} \mathrm{O}(20 \mathrm{~mL})$, filtered and dried in vacuo. The solid was redissolved in MeCN $(5 \mathrm{~mL})$, stirred for $30 \mathrm{~min}$ and then concentrated to dryness again. The resulting solid was dissolved in $\mathrm{CH}_{2} \mathrm{Cl}_{2}(10 \mathrm{~mL})$ and $n$-hexane $(100 \mathrm{~mL})$ was added to precipitate an off-white solid. Yield: $1.06 \mathrm{~g}, 70 \%$. Crystals were grown by layering a concentrated $\mathrm{CH}_{2} \mathrm{Cl}_{2}$ solution with $n$-hexane. Attempts to obtain accurate elemental analyses failed due to ready loss of $\mathrm{MeCN}$ ligands on drying. Samples sent for elemental analysis were consistent with between five and six MeCN ligands coordinated to calcium. The composition with eight MeCN ligands comes from the X-ray structure. ${ }^{1} \mathrm{H}$ NMR $\left(\mathrm{CD}_{2} \mathrm{Cl}_{2}\right.$, $298 \mathrm{~K}): \delta=7.72\left(\mathrm{~s}, \mathrm{BAr}^{\mathrm{F}} \mathrm{H} 2 / 6\right), 7.57\left(\mathrm{~s}, \mathrm{BAr}^{\mathrm{F}} \mathrm{H} 4\right), 2.07\left(\mathrm{~s}, \mathrm{CH}_{3}\right)$. IR (Nujol/ $\mathrm{cm}^{-1}$ ): 2310, 2282 (MeCN), 1611, 1282, 1118, 933, 897, 889, 840, 745, 713, 683, 671, 451.

$\left[\mathrm{Sr}(\mathbf{a c a c H})(\mathrm{MeCN})_{5}\right]\left[\mathrm{BAr}^{\mathrm{F}}\right]_{2} \cdot\left[\mathrm{Sr}(\mathrm{acac})_{2}\right](0.071 \mathrm{~g}, 0.25 \mathrm{mmol})$ was suspended in MeCN $(20 \mathrm{~mL})$ and a solution of $\left[\mathrm{H}\left(\mathrm{OEt}_{2}\right)_{2}\right]\left[\mathrm{BAr}^{\mathrm{F}}\right](0.51 \mathrm{~g}, 0.50 \mathrm{mmol})$ in $\mathrm{MeCN}(10 \mathrm{~mL})$ was 
Table 1 Crystallographic parameters for the compounds reported

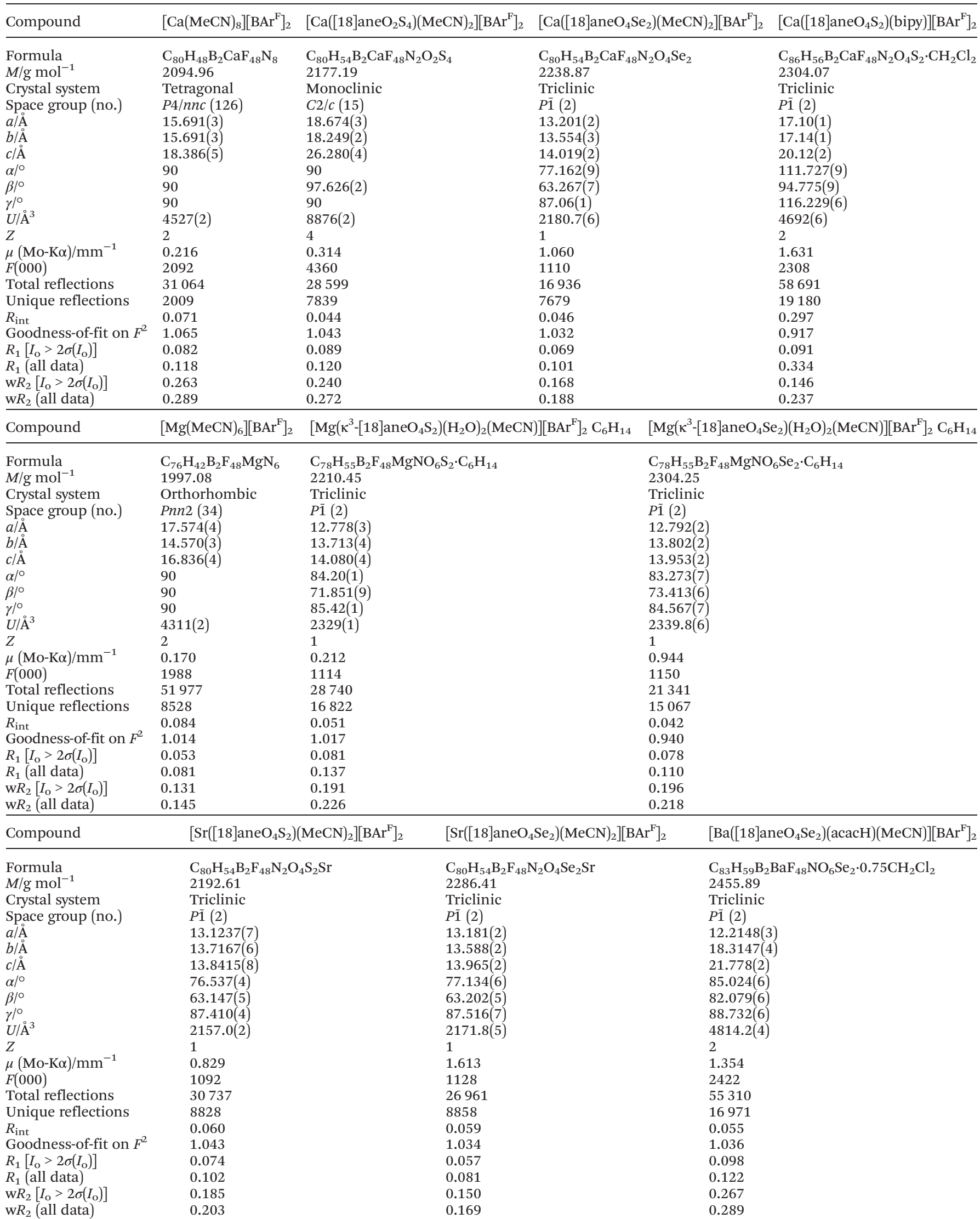

Common items: $T=100 \mathrm{~K}, \lambda_{1}=0.71073 \AA\left(\mathrm{Mo} \mathrm{K}_{\alpha}\right), \theta_{\max }=27.5^{\circ}, R_{1}=\sum|| F_{\mathrm{o}}|-| F_{\mathrm{c}}|| / \sum\left|F_{\mathrm{o}}\right|, \mathrm{w} R_{2}=\left[\sum \mathrm{w}\left(F_{\mathrm{o}}{ }^{2}-F_{\mathrm{c}}{ }^{2}\right)^{2} / \sum \mathrm{w} F_{\mathrm{o}}\right]^{1 / 2}$. 
added via a cannula. Most of the solids dissolved and the reaction was stirred for $16 \mathrm{~h}$. After this time solvents were removed and the resulting white solid extracted into $\mathrm{CH}_{2} \mathrm{Cl}_{2}(2 \times$ $30 \mathrm{~mL}$ ). The extracts were combined and concentrated to $\sim 5 \mathrm{~mL}$, then $n$-hexane $(30 \mathrm{~mL})$ was added to precipitate a white solid which was isolated by decanting away the supernatant and drying in vacuo. Yield: $0.45 \mathrm{~g}, 85 \% .{ }^{1} \mathrm{H}$ NMR $\left(\mathrm{CD}_{2} \mathrm{Cl}_{2}, 298 \mathrm{~K}\right): \delta=7.72\left(\mathrm{~s},[16 \mathrm{H}], \mathrm{BAr}^{\mathrm{F}} \mathrm{H} 2 / 6\right), 7.57(\mathrm{~s},[8 \mathrm{H}]$, $\left.\mathrm{BAr}^{\mathrm{F}} \mathrm{H} 4\right), 3.81$ (s, [2H], $\left.\mathrm{CH}_{2}, \mathrm{acacH}\right), 2.26\left(\mathrm{~s},[6 \mathrm{H}], \mathrm{CH}_{3}, \mathrm{acacH}\right)$, 2.13 (s, [15H], $\left.\mathrm{CH}_{3}, \mathrm{MeCN}\right) .{ }^{13} \mathrm{C}\left\{{ }^{1} \mathrm{H}\right\}$ NMR $\left(\mathrm{CD}_{2} \mathrm{Cl}_{2}, 298 \mathrm{~K}\right): \delta=$ $162.32\left(\mathrm{q}, J_{\mathrm{C}-\mathrm{B}}=49.9 \mathrm{~Hz}, \mathrm{BAr}^{\mathrm{F}}, \mathrm{C} 1\right), 135.38\left(\mathrm{BAr}^{\mathrm{F}}, \mathrm{C} 2 / 6\right), 129.47$ $\left(\mathrm{qq},{ }^{2} J_{\mathrm{C}-\mathrm{F}}=31.6,2.9 \mathrm{~Hz}, \mathrm{BAr}^{\mathrm{F}}, \mathrm{C} 3 / 5\right), 125.19\left(\mathrm{q}, J_{\mathrm{C}-\mathrm{F}}=272 \mathrm{~Hz}\right.$, $\mathrm{CF}_{3}$ ), 118.09 (septet, $\left.{ }^{3} J_{\mathrm{C}-\mathrm{F}}=4.0 \mathrm{~Hz}, \mathrm{BAr}^{\mathrm{F}}, \mathrm{C} 4\right), 52.89\left(\mathrm{CH}_{2}\right.$, acacH), $32.66\left(\mathrm{CH}_{3}, \mathrm{acacH}\right), 2.14\left(\mathrm{CH}_{3}, \mathrm{MeCN}\right)$. IR (Nujol/ $\mathrm{cm}^{-1}$ ): 2309 and 2281 (MeCN), 1751, 1714 (acacH) 1611, 1281, 1117, 932, 888, 840, 714, 683, 670. Despite repeated attempts, satisfactory elemental analysis could not be obtained due to the easy loss of the MeCN ligands. A few crystals of $\left[\mathrm{Sr}(\mathrm{MeCN})_{8}\right]\left[\mathrm{BAr}^{\mathrm{F}}\right]_{2}$ were obtained by layering a concentrated $\mathrm{CH}_{2} \mathrm{Cl}_{2}$ solution of the title compound with $n$-hexane.

$\left[\mathrm{Sr}(\mathbf{M e C N})_{8}\right]\left[\mathbf{B A r}^{\mathrm{F}}\right]_{2}$. Obtained as a by-product as above: ${ }^{1} \mathrm{H}$ NMR $\left(\mathrm{CD}_{2} \mathrm{Cl}_{2}, 298 \mathrm{~K}\right): \delta=7.72\left(\mathrm{~s},[16 \mathrm{H}], \mathrm{BAr}^{\mathrm{F}} \mathrm{H} 2 / 6\right), 7.58$ (s, [8H], BAr $\left.{ }^{\mathrm{F}} \mathrm{H} 4\right), 2.03$ (s, [24H], $\left.\mathrm{CH}_{3}, \mathrm{MeCN}\right) .{ }^{13} \mathrm{C}\left\{{ }^{1} \mathrm{H}\right\} \mathrm{NMR}$ $\left(\mathrm{CD}_{2} \mathrm{Cl}_{2}, 298 \mathrm{~K}\right): \delta=162.06$ (q, $\left.J_{\mathrm{C}-\mathrm{B}}=49.9 \mathrm{~Hz}, \mathrm{BAr}^{\mathrm{F}} \mathrm{C} 1\right), 135.37$ $\left(\mathrm{BAr}^{\mathrm{F}} \mathrm{C} 2 / 6\right), 129.64$ (qq, $\left.{ }^{2} J_{\mathrm{C}-\mathrm{F}}=31.6,2.9 \mathrm{~Hz}, \mathrm{BAr}^{\mathrm{F}} \mathrm{C} 3 / 5\right), 125.53$ (q, ${ }^{1} J_{\mathrm{C}-\mathrm{F}}=272 \mathrm{~Hz}, \mathrm{CF}_{3}$ ), 118.05 (septet, ${ }^{3} J_{\mathrm{C}-\mathrm{F}}=4.0 \mathrm{~Hz}, \mathrm{BAr}^{\mathrm{F}} \mathrm{C} 4$ ), 2.20 (s, $\left.\mathrm{CH}_{3}, \mathrm{MeCN}\right)$. IR (Nujol/cm ${ }^{-1}$ ): 2309, 2281 (MeCN), 1611, 1282, 1126, 888, 816, 683, 670.

$\left[\mathrm{Ba}(\mathbf{a c a c H})(\mathbf{M e C N})_{5}\right]\left[\mathrm{BAr}^{\mathrm{F}}\right]_{2}$. As for the $\mathrm{Sr}$ analogue, using [Ba(acac $\left.)_{2}\right](0.084 \mathrm{~g})$. Yield: $0.33 \mathrm{~g}$ of a white solid, $60 \%$. Anal. Required for $\mathrm{C}_{79} \mathrm{H}_{47} \mathrm{~B}_{2} \mathrm{BaF}_{48} \mathrm{~N}_{5} \mathrm{O}_{2}$ (2169.49): C, 43.74; H, 2.18; $\mathrm{N}, 3.23$. Found: $\mathrm{C}, 43.63 ; \mathrm{H}, 2.04 ; \mathrm{N}, 3.35 \%$. ${ }^{1} \mathrm{H}$ NMR $\left(\mathrm{CD}_{2} \mathrm{Cl}_{2}\right.$, $298 \mathrm{~K}): \delta=7.72\left(\mathrm{~s},[16 \mathrm{H}], \mathrm{BAr}^{\mathrm{F}} \mathrm{H} 2 / 6\right), 7.58\left(\mathrm{~s},[8 \mathrm{H}], \mathrm{BAr}^{\mathrm{F}} \mathrm{H} 4\right)$, $2.67\left(\mathrm{~s},[6 \mathrm{H}]\right.$, acacH $\left.\mathrm{CH}_{3}\right), 2.24$ and 2.20 (each $\mathrm{s},[3 \mathrm{H}]$, acacH $\mathrm{CH}_{2}$ ), 2.00 (s, [15H], $\left.\mathrm{CH}_{3} \mathrm{MeCN}\right) .{ }^{13} \mathrm{C}\left\{{ }^{1} \mathrm{H}\right\} \mathrm{NMR}\left(\mathrm{CD}_{2} \mathrm{Cl}_{2}\right.$, $298 \mathrm{~K}): \delta=162.32\left(\mathrm{q}, J_{\mathrm{C}-\mathrm{B}}=49.9 \mathrm{~Hz}, \mathrm{BAr}^{\mathrm{F}} \mathrm{C} 1\right), 135.38$ $\left(\mathrm{BAr}^{\mathrm{F}} \mathrm{C} 2 / 6\right), 129.48$ (qq, $\left.{ }^{2} J_{\mathrm{C}-\mathrm{F}}=31.6,2.9 \mathrm{~Hz}, \mathrm{BAr}^{\mathrm{F}} \mathrm{C} 3 / 5\right), 125.19$ (q, ${ }^{1} J_{\mathrm{C}-\mathrm{F}}=272 \mathrm{~Hz}, \mathrm{CF}_{3}$ ), 118.10 (septet, ${ }^{3} J_{\mathrm{C}-\mathrm{F}}=4.0 \mathrm{~Hz}, \mathrm{BAr}^{\mathrm{F}} \mathrm{C} 4$ ), $80.60\left(\mathrm{CH}_{2}\right.$, acacH $), 32.65\left(\mathrm{CH}_{3}, \mathrm{acacH}\right), 1.98\left(\mathrm{CH}_{3}, \mathrm{MeCN}\right)$. IR (Nujol/cm ${ }^{-1}$ ): 2307, 2277 (MeCN), 1751 (acacH), 1611, 1281, 1117, 888, 840, 713, 683, 670 .

\section{General method for complex synthesis}

The Group 2 [BAr $\left.{ }^{\mathrm{F}}\right]$ salt was suspended in $\mathrm{CH}_{2} \mathrm{Cl}_{2}(5 \mathrm{~mL})$ and a solution of the macrocycle in $\mathrm{CH}_{2} \mathrm{Cl}_{2}(5 \mathrm{~mL})$ was added. Complete dissolution occurred and the reaction was stirred for $16 \mathrm{~h}$. After this time the solution was filtered, concentrated to $\sim 3 \mathrm{~mL}$ and layered with $n$-hexane $(20 \mathrm{~mL})$ to form crystals. These were isolated by decanting away the supernatant and drying the solid in vacuo.

$\left[\mathbf{M g}(\mathbf{1 8 - c r o w n}-6)(\mathbf{M e C N})_{2}\right]\left[\mathrm{BAr}^{\mathrm{F}}\right]_{2} \cdot\left[\mathrm{Mg}(\mathrm{MeCN})_{6}\right]\left[\mathrm{BAr}^{\mathrm{F}}\right]_{2}(0.050 \mathrm{~g}$, $0.025 \mathrm{mmol})$ and 18-crown-6 $(0.007 \mathrm{~g}, 0.025 \mathrm{mmol})$ afforded $0.039 \mathrm{~g}$ of a yellow solid in $74 \%$ yield. Anal. Required for $\mathrm{C}_{80} \mathrm{H}_{54} \mathrm{~B}_{2} \mathrm{~F}_{48} \mathrm{MgN}_{2} \mathrm{O}_{6}$ (2097.19): C, 45.82; H, 2.60; N, 1.34. Found: C, 45.64; H, 2.50; N, 1.26\%. ${ }^{1} \mathrm{H}$ NMR $\left(\mathrm{CD}_{2} \mathrm{Cl}_{2}, 298 \mathrm{~K}\right)$ : $\delta=7.73\left(\mathrm{~s},[16 \mathrm{H}], \mathrm{BAr}^{\mathrm{F}} \mathrm{H} 2 / 6\right), 7.58\left(\mathrm{~s},[8 \mathrm{H}], \mathrm{BAr}^{\mathrm{F}} \mathrm{H} 4\right), 3.85$ (s, [24H], $\left.\mathrm{OCH}_{2}\right), 2.15$ (s, [6H] $\left., \mathrm{CH}_{3}, \mathrm{MeCN}\right) .{ }^{13} \mathrm{C}\left\{{ }^{1} \mathrm{H}\right\} \mathrm{NMR}$ $\left(\mathrm{CD}_{2} \mathrm{Cl}_{2}, 298 \mathrm{~K}\right): \delta=162.31\left(\mathrm{q},{ }^{1} J_{\mathrm{C}-\mathrm{B}}=50.0 \mathrm{~Hz}, \mathrm{BAr}^{\mathrm{F}} \mathrm{C} 1\right), 135.40$ $\left(\mathrm{BAr}^{\mathrm{F}} \mathrm{C} 2 / 6\right) 129.48\left(\mathrm{qq},{ }^{2} J_{\mathrm{C}-\mathrm{F}}=31.6,2.9 \mathrm{~Hz} \mathrm{BAr}^{\mathrm{F}}, \mathrm{C} 3 / 5\right), 125.18$ $\left(\mathrm{q},{ }^{1} J_{\mathrm{C}-\mathrm{F}}=272 \mathrm{~Hz}, \mathrm{CF}_{3}\right), 119.33(\mathrm{C}, \mathrm{MeCN}), 118.16$ (septet, $\left.{ }^{3} J_{\mathrm{C}-\mathrm{F}}=4.0 \mathrm{~Hz}, \mathrm{BAr}^{\mathrm{F}}, \mathrm{C} 4\right) 70.02\left(\mathrm{~s}, \mathrm{OCH}_{2}\right) 2.61\left(\mathrm{CH}_{3}, \mathrm{MeCN}\right)$. IR (Nujol/cm ${ }^{-1}$ ): 2316, 2289 (MeCN), 1611, 1280, 1122 1106, 889, 839, 802, 682, 670, 402.

$\left[\mathbf{M g}\left([\mathbf{1 8}] \operatorname{aneO}_{4} \mathbf{S}_{2}\right)(\mathbf{M e C N})_{2}\right]\left[\mathbf{B A r}^{\mathrm{F}}\right]_{2} \cdot\left[\mathrm{Mg}(\mathrm{MeCN})_{6}\right]\left[\mathrm{BAr}^{\mathrm{F}}\right]_{2}$ $(0.050 \mathrm{~g}, 0.025 \mathrm{mmol})$ and $[18] \mathrm{aneO}_{4} \mathrm{~S}_{2}(0.007 \mathrm{~g}, 0.025 \mathrm{mmol})$ afforded $0.044 \mathrm{~g}$ of an off-white solid in $82 \%$ yield. Anal. Required for $\mathrm{C}_{80} \mathrm{H}_{54} \mathrm{~B}_{2} \mathrm{~F}_{48} \mathrm{MgN}_{2} \mathrm{O}_{4} \mathrm{~S}_{2} \cdot \mathrm{CH}_{2} \mathrm{Cl}_{2} \quad$ (2214.07): C, 43.94; H, 2.55; N, 1.26. Found: C, $43.67 \mathrm{H}, 2.74$; N, 1.06\%. ${ }^{1} \mathrm{H}$ NMR $\left(\mathrm{CD}_{2} \mathrm{Cl}_{2}, 298 \mathrm{~K}\right): \delta=7.75$ (br s, [16H], $\left.\mathrm{BAr}^{\mathrm{F}} \mathrm{H} 2 / 6\right), 7.60$ (s, [8H], BAr ${ }^{\mathrm{F}} \mathrm{H} 4$ ), 3.93 (br s, [16H], $\mathrm{OCH}_{2}$ ), 2.98 (br, t, $[8 \mathrm{H}]$, $\left.\mathrm{SCH}_{2}\right), 2.19$ (s, [6H], $\left.\mathrm{CH}_{3} \mathrm{MeCN}\right) .{ }^{13} \mathrm{C}\left\{{ }^{1} \mathrm{H}\right\} \mathrm{NMR}\left(\mathrm{CD}_{2} \mathrm{Cl}_{2}, 298 \mathrm{~K}\right)$ : $\delta=161.93\left(\mathrm{q},{ }^{1} J_{\mathrm{C}-\mathrm{B}}=50.0 \mathrm{~Hz}, \mathrm{BAr}^{\mathrm{F}} \mathrm{C} 1\right), 135.96\left(\mathrm{BAr}^{\mathrm{F}} \mathrm{C} 2 / 6\right)$, $129.06\left(\mathrm{qq},{ }^{2} J_{\mathrm{C}-\mathrm{F}}=31.6,2.9 \mathrm{~Hz}, \mathrm{BAr}^{\mathrm{F}}, \mathrm{C} 3 / 5\right), 124.78\left(\mathrm{q},{ }^{1} J_{\mathrm{C}-\mathrm{F}}=\right.$ $\left.272 \mathrm{~Hz}, \mathrm{CF}_{3}\right), 118.16\left(\mathrm{~s},{ }^{3} J_{\mathrm{CF}}=4.0 \mathrm{~Hz}, \mathrm{BAr}^{\mathrm{F}}, \mathrm{C} 4\right), 117.72$ (C, MeCN), 69.07 (s, $\left.\mathrm{OCH}_{2}\right), 31.24\left(\mathrm{~s}, \mathrm{SCH}_{2}\right), 2.14\left(\mathrm{CH}_{3}, \mathrm{MeCN}\right)$. IR (Nujol/cm $\mathrm{cm}^{-1}$ ): 2317, 2294 (MeCN), 1611, 1277, 1094, 1019, $887,801,682,669$.

$\left[\mathbf{M g}\left([\mathbf{1 8}]\right.\right.$ aneO $\left._{2} \mathbf{S}_{4}\right)\left(\mathbf{M e C N}_{2}\right]\left[\mathbf{B A r}^{\mathrm{F}}\right]_{2} \cdot\left[\mathrm{Mg}(\mathrm{MeCN})_{6}\right]\left[\mathrm{BAr}^{\mathrm{F}}\right]_{2}$ $(0.050 \mathrm{~g}, 0.025 \mathrm{mmol})$ and $[18] \mathrm{aneO}_{2} \mathrm{~S}_{4}(0.008 \mathrm{~g}, 0.025 \mathrm{mmol})$ afforded $0.034 \mathrm{~g}$ of an off-white solid in $63 \%$ yield. Anal. Required for $\mathrm{C}_{80} \mathrm{H}_{54} \mathrm{~B}_{2} \mathrm{~F}_{48} \mathrm{MgN}_{2} \mathrm{O}_{2} \mathrm{~S}_{4}$ (2161.09): $\mathrm{C}, 44.46$; $\mathrm{H}, 2.52$; N, 1.30. Found: C, 44.61; H, 2.38; N, 1.32\%. ${ }^{1} \mathrm{H}$ NMR $\left(\mathrm{CD}_{2} \mathrm{Cl}_{2}, 298 \mathrm{~K}\right): \delta=7.74\left(\mathrm{~s}, \mathrm{BAr}^{\mathrm{F}} \mathrm{H} 2 / 6\right), 7.58\left(\mathrm{~s}, \mathrm{BAr}^{\mathrm{F}} \mathrm{H} 4\right), 3.78$ (vbr, s, $\mathrm{OCH}_{2}$ ), 2.84-2.94 (br s, $\mathrm{SCH}_{2}$ ), 2.42 (s, $\mathrm{CH}_{3}, \mathrm{MeCN}$ ). ${ }^{13} \mathrm{C}\left\{{ }^{1} \mathrm{H}\right\} \operatorname{NMR}\left(\mathrm{CD}_{2} \mathrm{Cl}_{2}, 298 \mathrm{~K}\right): \delta=162.13\left(\mathrm{q},{ }^{1} J_{\mathrm{C}-\mathrm{B}}=50.0 \mathrm{~Hz}\right.$, $\left.\mathrm{BAr}^{\mathrm{F}} \mathrm{C} 1\right), 134.88\left(\mathrm{BAr}^{\mathrm{F}} \mathrm{C} 2 / 6\right), 129.16\left(\mathrm{br} \mathrm{q},{ }^{2} J_{\mathrm{C}-\mathrm{F}}=31.6 \mathrm{~Hz}\right.$, $\left.\mathrm{BAr}^{\mathrm{F}} \mathrm{C} 3 / 5\right), 124.78\left(\mathrm{q},{ }^{1} J_{\mathrm{C}-\mathrm{F}}=272 \mathrm{~Hz}, \mathrm{CF}_{3}\right), 118.16\left(\mathrm{BAr}^{\mathrm{F}} \mathrm{C} 4\right)$, 117.93 (C, MeCN), $1.84\left(\mathrm{CH}_{3}, \mathrm{MeCN}\right)$ - see text. IR (Nujol $\left./ \mathrm{cm}^{-1}\right)$ : 2322, 2296 (MeCN), 1612, 1282, 1115, 1023, 889, 840, 683, 670.

$\left[\mathrm{Mg}\left([\mathbf{1 8}] \mathrm{aneO}_{4} \mathrm{Se}_{2}\right)\left(\mathrm{MeCN}_{2}\right]\left[\mathrm{BAr}^{\mathrm{F}}\right]_{2} \cdot\left[\mathrm{Mg}(\mathrm{MeCN})_{6}\right]\left[\mathrm{BAr}^{\mathrm{F}}\right]_{2}\right.$ $(0.075 \mathrm{~g}, 0.038 \mathrm{mmol})$ and $[18] \mathrm{aneO}_{4} \mathrm{Se}_{2}(0.015 \mathrm{~g}, 0.038 \mathrm{mmol})$ afforded $0.057 \mathrm{~g}$ of a white powdery solid in $68 \%$ yield. Anal. Required for $\mathrm{C}_{80} \mathrm{H}_{54} \mathrm{~B}_{2} \mathrm{~F}_{48} \mathrm{MgN}_{2} \mathrm{O}_{4} \mathrm{Se}_{2}$ (2223.12): C, 43.22; $\mathrm{H}, 2.45$; N, 1.26. Found: C, 43.16; H, 2.44; N, 1.35\%. ${ }^{1} \mathrm{H}$ NMR $\left(\mathrm{CD}_{2} \mathrm{Cl}_{2}, 298 \mathrm{~K}\right): \delta=7.75$ (br s, $\left.[16 \mathrm{H}], \mathrm{BAr}^{\mathrm{F}} \mathrm{H} 2 / 6\right), 7.60(\mathrm{~s},[8 \mathrm{H}]$, $\left.\mathrm{BAr}^{\mathrm{F}} \mathrm{H} 4\right), 4.01$ (br, s, [16H], $\mathrm{OCH}_{2}$ ), 2.95 (br s, [8H], $\mathrm{SeCH}_{2}$ ), 2.15 (s, [6H], MeCN). ${ }^{13} \mathrm{C}\left\{{ }^{1} \mathrm{H}\right\} \mathrm{NMR}\left(\mathrm{CD}_{2} \mathrm{Cl}_{2}, 298 \mathrm{~K}\right): \delta=162.13$ $\left(\mathrm{q},{ }^{1} J_{\mathrm{C}-\mathrm{B}}=50.0 \mathrm{~Hz}, \mathrm{BAr}^{\mathrm{F}} \mathrm{C} 1\right), 135.09\left(\mathrm{BAr}^{\mathrm{F}} \mathrm{C} 2 / 6\right), 129.06$ $\left(\mathrm{qq},{ }^{2} J_{\mathrm{C}-\mathrm{F}}=31.6,2.9 \mathrm{~Hz}, \mathrm{BAr}{ }^{\mathrm{F}}, \mathrm{C} 3 / 5\right), 124.78\left(\mathrm{q},{ }^{1} J_{\mathrm{C}-\mathrm{F}}=272 \mathrm{~Hz}\right.$, $\mathrm{CF}_{3}$ ), 118.16 (septet, ${ }^{3} J_{C-F}=4.0 \mathrm{~Hz}, \mathrm{BAr}^{\mathrm{F}}, \mathrm{C} 4$ ), 117.78 (C, MeCN), $68.85\left(\mathrm{~s}, \mathrm{OCH}_{2}\right), 23.47\left(\mathrm{~s}, \mathrm{SeCH}_{2}\right), 2.04\left(\mathrm{CH}_{3}\right.$, $\mathrm{MeCN}$ ). IR (Nujol/cm ${ }^{-1}$ ): 2322, 2295 (MeCN), 1611, 1281, 1112, 888, 839, 683, 669.

$\left[\mathrm{Ca}(18-\text { crown-6)(MeCN })_{2}\right]\left[\mathrm{BAr}^{\mathrm{F}}\right]_{2} \cdot\left[\mathrm{Ca}(\mathrm{MeCN})_{8}\right]\left[\mathrm{BAr}^{\mathrm{F}}\right]_{2}(0.100 \mathrm{~g}$, $0.048 \mathrm{mmol})$ and 18-crown-6 $(0.013 \mathrm{~g}, 0.048 \mathrm{mmol})$ afforded $0.078 \mathrm{~g}$ of a white solid in $77 \%$ yield. Anal. Required for $\mathrm{C}_{80} \mathrm{H}_{54} \mathrm{~B}_{2} \mathrm{CaF}_{48} \mathrm{~N}_{2} \mathrm{O}_{6}$ (2113.28): C, 45.48; H, 2.58; N, 1.33. Found: C, 45.55; H, 2.53; N, 1.41\%. ${ }^{1} \mathrm{H} \mathrm{NMR}\left(\mathrm{CD}_{2} \mathrm{Cl}_{2}, 298 \mathrm{~K}\right)$ : $\delta=7.73\left(\mathrm{~s},[16 \mathrm{H}], \mathrm{BAr}^{\mathrm{F}} \mathrm{H} 2 / 6\right), 7.57\left(\mathrm{~s},[8 \mathrm{H}], \mathrm{BAr}^{\mathrm{F}} \mathrm{H} 4\right), 3.81$ $\left(\mathrm{s},[18 \mathrm{H}], \mathrm{OCH}_{2}\right), 2.13\left(\mathrm{~s},[6 \mathrm{H}], \mathrm{CH}_{3}, \mathrm{MeCN}\right) .{ }^{13} \mathrm{C}\left\{{ }^{1} \mathrm{H}\right\} \mathrm{NMR}$ $\left(\mathrm{CD}_{2} \mathrm{Cl}_{2}, 298 \mathrm{~K}_{2}\right): \delta=162.31\left(\mathrm{q}, J_{\mathrm{C}-\mathrm{B}}=49.9 \mathrm{~Hz}, \mathrm{BAr}^{\mathrm{F}} \mathrm{C} 1\right), 135.37$ $\left(\mathrm{BAr}^{\mathrm{F}} \mathrm{C} 2 / 6\right), 129.45$ (qq, $\left.^{2} J_{C-F}=31.6,2.9 \mathrm{~Hz}, \mathrm{BAr}^{\mathrm{F}} \mathrm{C} 3 / 5\right), 125.17$ 
$\left(\mathrm{q}, J_{C-F}=272 \mathrm{~Hz}, \mathrm{CF}_{3}\right), 121.11(\mathrm{C}, \mathrm{MeCN}), 118.08$ (septet, $\left.{ }^{3} J_{C-F}=4.0 \mathrm{~Hz}, \mathrm{BAr}^{\mathrm{F}} \mathrm{C} 4\right), 70.04\left(\mathrm{OCH}_{2}\right), 2.29\left(\mathrm{CH}_{3}, \mathrm{MeCN}\right)$. IR (Nujol/cm ${ }^{-1}$ ): 2309, 2279 (MeCN), 1611, 1280, 1129, 967, 901, 840, 682, 671, 586, 450.

$\left[\mathrm{Ca}\left([18] \operatorname{aneO}_{4} \mathbf{S}_{2}\right)(\mathbf{M e C N})_{2}\right]\left[\mathrm{BAr}^{\mathrm{F}}\right]_{2} \cdot\left[\mathrm{Ca}(\mathrm{MeCN})_{8}\right]\left[\mathrm{BAr}^{\mathrm{F}}\right]_{2}(0.100 \mathrm{~g}$, $0.048 \mathrm{mmol})$ and [18] $\mathrm{aneO}_{4} \mathrm{~S}_{2}(0.014 \mathrm{~g}, 0.048 \mathrm{mmol})$ afforded $0.078 \mathrm{~g}$ of a white solid in $76 \%$ yield. Anal. Required for $\mathrm{C}_{80} \mathrm{H}_{54} \mathrm{~B}_{2} \mathrm{CaF}_{48} \mathrm{~N}_{2} \mathrm{O}_{4} \mathrm{~S}_{2}$ (2145.23): C, 44.79; H, 2.54; N, 1.31. Found: C, 44.63; H, 2.45; N, 1.27\%. ${ }^{1} \mathrm{H} \mathrm{NMR}\left(\mathrm{CD}_{2} \mathrm{Cl}_{2}, 298 \mathrm{~K}\right)$ : $\delta=7.73\left(\mathrm{~s},[16 \mathrm{H}], \mathrm{BAr}^{\mathrm{F}} \mathrm{H} 2 / 6\right), 7.57$ (s, [8H], $\left.\mathrm{BAr}^{\mathrm{F}} \mathrm{H} 4\right), 3.86-3.89$ $\left(\mathrm{m},[16 \mathrm{H}], \mathrm{OCH}_{2}\right), 2.89$ (br s, $\left.[8 \mathrm{H}], \mathrm{SCH}_{2}\right), 2.10\left(\mathrm{~s},[6 \mathrm{H}], \mathrm{CH}_{3}\right.$, MeCN). ${ }^{13} \mathrm{C}\left\{{ }^{1} \mathrm{H}\right\} \operatorname{NMR}\left(\mathrm{CD}_{2} \mathrm{Cl}_{2}, 298 \mathrm{~K}\right): \delta=162.33$ (q, $J_{\mathrm{C}-\mathrm{B}}=$ $\left.49.9 \mathrm{~Hz}, \mathrm{BAr}^{\mathrm{F}} \mathrm{C} 1\right), 135.39\left(\mathrm{BAr}^{\mathrm{F}} \mathrm{C} 2 / 6\right), 129.48\left(\mathrm{qq},{ }^{2} J_{\mathrm{C}-\mathrm{F}}=31.6\right.$, $\left.2.9 \mathrm{~Hz}, \mathrm{BAr}^{\mathrm{F}} \mathrm{C} 3 / 5\right), 125.19\left(\mathrm{q}, J_{\mathrm{C}-\mathrm{F}}=272 \mathrm{~Hz}, \mathrm{CF}_{3}\right), 119.06$ (C, MeCN), 118.11 (septet, ${ }^{3} J_{\mathrm{C}-\mathrm{F}}=4.0 \mathrm{~Hz}, \mathrm{BAr}^{\mathrm{F}} \mathrm{C} 4$ ), 70.95, 70.70 $\left(\mathrm{OCH}_{2}\right), 31.73\left(\mathrm{SCH}_{2}\right), 2.30\left(\mathrm{CH}_{3}, \mathrm{MeCN}\right) . \mathrm{IR}\left(\mathrm{Nujol} / \mathrm{cm}^{-1}\right)$ : 2307, 2276 (MeCN), 1611, 1279, 1117, 901, 889, 839, 744, 682, $670,449$.

$\left[\mathrm{Ca}\left([\mathbf{1 8}] \mathbf{a n e O}_{2} \mathbf{S}_{4}\right)(\mathbf{M e C N})_{2}\right]\left[\mathrm{BAr}^{\mathrm{F}}\right]_{2} \cdot\left[\mathrm{Ca}(\mathrm{MeCN})_{8}\right]\left[\mathrm{BAr}^{\mathrm{F}}\right]_{2}(0.100 \mathrm{~g}$, $0.048 \mathrm{mmol})$ and $[18] \mathrm{aneO}_{2} \mathrm{~S}_{4}(0.016 \mathrm{~g}, 0.048 \mathrm{mmol})$ afforded $0.074 \mathrm{~g}$ of a white solid in $71 \%$ yield. Anal. Required for $\mathrm{C}_{80} \mathrm{H}_{54} \mathrm{~B}_{2} \mathrm{CaF}_{48} \mathrm{~N}_{2} \mathrm{O}_{2} \mathrm{~S}_{4}$ (2177.19): C, 44.13; H, 2.50; N, 1.29. Found: C, 43.91; H, 2.41; N, 1.38\%. ${ }^{1} \mathrm{H}$ NMR $\left(\mathrm{CD}_{2} \mathrm{Cl}_{2}, 298 \mathrm{~K}\right)$ : $\delta=7.72\left(\mathrm{~s},[16 \mathrm{H}], \mathrm{BAr}^{\mathrm{F}} \mathrm{H} 2 / 6\right), 7.58\left(\mathrm{~s},[8 \mathrm{H}], \mathrm{BAr}^{\mathrm{F}} \mathrm{H} 4\right), 3.90(\mathrm{t}$, $\left.J=5.2 \mathrm{~Hz},[8 \mathrm{H}], \mathrm{OCH}_{2}\right), 3.01\left(\mathrm{~s},[8 \mathrm{H}], \mathrm{SCH}_{2}\right), 2.92(\mathrm{t}, J=5.0 \mathrm{~Hz}$, $\left.[8 \mathrm{H}], \mathrm{SCH}_{2}\right), 2.13$ (s, [6H], $\left.\mathrm{CH}_{3} \mathrm{MeCN}\right) .{ }^{13} \mathrm{C}\left\{{ }^{1} \mathrm{H}\right\} \mathrm{NMR}\left(\mathrm{CD}_{2} \mathrm{Cl}_{2}\right.$, $298 \mathrm{~K}): \delta=162.34$ (q, $\left.J_{\mathrm{C}-\mathrm{B}}=49.9 \mathrm{~Hz}, \mathrm{BAr}^{\mathrm{F}} \mathrm{C} 1\right), 135.40\left(\mathrm{BAr}^{\mathrm{F}}\right.$ C2/6), 129.49 (qq, $\left.{ }^{2} J_{\mathrm{C}-\mathrm{F}}=31.6,2.9 \mathrm{~Hz}, \mathrm{BAr}^{\mathrm{F}} \mathrm{C} 3 / 5\right), 125.21$ (C, q, $\left.J_{\mathrm{C}-\mathrm{F}}=272 \mathrm{~Hz}, \mathrm{CF}_{3}\right), 120.25(\mathrm{C}, \mathrm{MeCN}), 118.12(\mathrm{CH}$, septet, $\left.{ }^{3} J_{\mathrm{C}-\mathrm{F}}=4.0 \mathrm{~Hz}, \mathrm{BAr}{ }^{\mathrm{F}} \mathrm{C} 4\right), 70.97\left(\mathrm{OCH}_{2}\right), 32.12,31.99\left(\mathrm{SCH}_{2}\right)$, $2.57\left(\mathrm{CH}_{3}, \mathrm{MeCN}\right)$. IR (Nujol/cm $\left.{ }^{-1}\right): 2307,2275$ (MeCN), 1611, 1279, 1120, 901, 839, 682, 671.

$\left[\mathrm{Ca}\left([18]\right.\right.$ aneO $\left._{4} \mathrm{Se}_{2}\right)\left(\mathrm{MeCN}_{2}\right]\left[\mathrm{BAr}^{\mathrm{F}}\right]_{2} \cdot\left[\mathrm{Ca}(\mathrm{MeCN})_{8}\right]\left[\mathrm{BAr}^{\mathrm{F}}\right]_{2}$ $(0.100 \mathrm{~g}, 0.048 \mathrm{mmol})$ and $[18] \mathrm{aneO}_{4} \mathrm{Se}_{2}(0.019 \mathrm{~g}, 0.048 \mathrm{mmol})$ afforded $0.077 \mathrm{~g}$ of a white solid in $72 \%$ yield. Anal. calc. for $\mathrm{C}_{80} \mathrm{H}_{54} \mathrm{~B}_{2} \mathrm{CaF}_{48} \mathrm{~N}_{2} \mathrm{O}_{4} \mathrm{Se}_{2}$ (2239.21): C, 42.91; H, 2.43; N, 1.25. Found: C, 42.82; H, 2.36; N, 1.16\%. ${ }^{1} \mathrm{H} \mathrm{NMR}\left(\mathrm{CD}_{2} \mathrm{Cl}_{2}, 298 \mathrm{~K}\right)$ : $\delta=7.72\left(\mathrm{~s},[16 \mathrm{H}], \mathrm{BAr}^{\mathrm{F}} \mathrm{H} 2 / 6\right), 7.58\left(\mathrm{~s},[8 \mathrm{H}], \mathrm{BAr}^{\mathrm{F}} \mathrm{H} 4\right), 3.95$ $\left(\mathrm{t}, J=5.6 \mathrm{~Hz},[8 \mathrm{H}], \mathrm{OCH}_{2}\right), 3.91\left(\mathrm{~s},[8 \mathrm{H}], \mathrm{OCH}_{2}\right), 2.87(\mathrm{br} \mathrm{s}$, $\left.[8 \mathrm{H}], \mathrm{SeCH}_{2}\right), 2.15$ (s, [6H], $\left.\mathrm{CH}_{3} \mathrm{MeCN}\right) .{ }^{13} \mathrm{C}\left\{{ }^{1} \mathrm{H}\right\} \mathrm{NMR}\left(\mathrm{CD}_{2} \mathrm{Cl}_{2}\right.$, $298 \mathrm{~K}): \delta=162.33$ (q, $\left.J_{\mathrm{C}-\mathrm{B}}=49.9 \mathrm{~Hz}, \mathrm{BAr}^{\mathrm{F}} \mathrm{C} 1\right), 135.39\left(\mathrm{BAr}^{\mathrm{F}}\right.$ $\mathrm{C} 2 / 6), 129.47\left(\mathrm{qq},{ }^{2} J_{\mathrm{C}-\mathrm{F}}=31.6,2.9 \mathrm{~Hz}, \mathrm{BAr}^{\mathrm{F}} \mathrm{C} 3 / 5\right), 125.20$ $\left(\mathrm{q}, J_{\mathrm{C}-\mathrm{F}}=272 \mathrm{~Hz}, \mathrm{CF}_{3}\right), 120.63(\mathrm{C}, \mathrm{MeCN}), 118.10$ (septet, $\left.{ }^{3} J_{\mathrm{C}-\mathrm{F}}=4.0 \mathrm{~Hz}, \mathrm{BAr}^{\mathrm{F}} \mathrm{C} 4\right), 71.45,71.32\left(\mathrm{OCH}_{2}\right), 25.53\left(\mathrm{SCH}_{2}\right)$, $2.63\left(\mathrm{CH}_{3}, \mathrm{MeCN}\right)$. IR (Nujol/cm $\left.{ }^{-1}\right): 2307,2275$ (MeCN), 1611, 1280, 1130, 903, 840, 671.

$\left[\operatorname{Sr}(18 \text {-crown-6)(MeCN })_{2}\right]\left[\mathbf{B A r}^{\mathrm{F}}\right]_{2} \cdot\left[\mathrm{Sr}(\mathrm{acacH})(\mathrm{MeCN})_{5}\right]\left[\mathrm{BAr}^{\mathrm{F}}\right]_{2}$ (0.100 g, $0.047 \mathrm{mmol})$ and 18-crown-6 (0.012 g, $0.047 \mathrm{mmol})$ afforded $0.069 \mathrm{~g}$ of a white solid in $68 \%$ yield. Anal. Required for $\mathrm{C}_{80} \mathrm{H}_{54} \mathrm{~B}_{2} \mathrm{~F}_{48} \mathrm{~N}_{2} \mathrm{O}_{6} \mathrm{Sr}$ (2160.82): C, 44.47; H, 2.52; N, 1.30. Found: C, 44.34; H, 2.38; N, 1.39\%. ${ }^{1} \mathrm{H} \mathrm{NMR}\left(\mathrm{CD}_{2} \mathrm{Cl}_{2}, 298 \mathrm{~K}\right)$ : $\delta=7.72\left(\right.$ br s, $\left.[16 \mathrm{H}], \mathrm{BAr}^{\mathrm{F}} \mathrm{H} 2 / 6\right), 7.58$ (s, [8H], BAr $\left.{ }^{\mathrm{F}} \mathrm{H} 4\right), 3.73$ (s, [24H], $\mathrm{OCH}_{2}$ ), 2.13 (s, [6H], $\left.\mathrm{CH}_{3} \mathrm{MeCN}\right) \cdot{ }^{13} \mathrm{C}\left\{{ }^{1} \mathrm{H}\right\} \mathrm{NMR}$ $\left(\mathrm{CD}_{2} \mathrm{Cl}_{2}, 298 \mathrm{~K}\right): \delta=162.31$ (q, $\left.J_{\mathrm{C}-\mathrm{B}}=49.9 \mathrm{~Hz}, \mathrm{BAr}^{\mathrm{F}} \mathrm{C} 1\right), 135.39$ $\left(\mathrm{BAr}^{\mathrm{F}} \mathrm{C} 2 / 6\right), 129.48\left(\mathrm{qq},{ }^{2} J_{\mathrm{C}-\mathrm{F}}=31.6,2.9 \mathrm{~Hz}, \mathrm{BAr}^{\mathrm{F}} \mathrm{C} 3 / 5\right)$, $125.18\left(\mathrm{q},{ }^{1} J_{\mathrm{C}-\mathrm{F}}=272 \mathrm{~Hz}, \mathrm{CF}_{3}\right), 119.33(\mathrm{C}, \mathrm{MeCN}), 118.10$ (septet, $\left.{ }^{3} J_{\mathrm{C}-\mathrm{F}}=4.0 \mathrm{~Hz}, \mathrm{BAr}^{\mathrm{F}} \mathrm{C} 4\right), 70.70\left(\mathrm{OCH}_{2}\right), 2.23\left(\mathrm{CH}_{3}\right.$, $\mathrm{MeCN}$ ). IR (Nujol/cm ${ }^{-1}$ ): 2308, 2278 (MeCN), 1611, 1282, 1120, 1092, 974, 932, 900, 886, 839, 745, 682, 670, 448, 366.

$\left[\mathrm{Sr}\left([\mathbf{1 8}] \mathbf{a n e O}_{4} \mathbf{S}_{2}\right)(\mathbf{M e C N})_{2}\right]\left[\mathrm{BAr}^{\mathrm{F}}\right]_{2} \cdot\left[\mathrm{Sr}(\mathrm{acacH})(\mathrm{MeCN})_{5}\right]\left[\mathrm{BAr}^{\mathrm{F}}\right]_{2}$ $(0.100 \mathrm{~g}, 0.047 \mathrm{mmol})$ and $[18] \mathrm{aneO}_{4} \mathrm{~S}_{2}(0.014 \mathrm{~g}, 0.047 \mathrm{mmol})$ afforded $0.089 \mathrm{~g}$ of a white solid in $86 \%$ yield. Anal. Required for $\mathrm{C}_{80} \mathrm{H}_{54} \mathrm{~B}_{2} \mathrm{~F}_{48} \mathrm{~N}_{2} \mathrm{O}_{4} \mathrm{~S}_{2} \mathrm{Sr}$ (2192.78): C, 43.82; H, 2.48; N, 1.28. Found: C, 43.69; H, 2.53; N, 1.25\%. ${ }^{1} \mathrm{H} \mathrm{NMR}\left(\mathrm{CD}_{2} \mathrm{Cl}_{2}, 298 \mathrm{~K}\right)$ : $\delta=7.73$ (br s, [16H], BAr $\left.{ }^{\mathrm{F}} \mathrm{H} 2 / 6\right), 7.58$ (s, [8H], $\left.\mathrm{BAr}^{\mathrm{F}} \mathrm{H} 4\right), 3.82$ $\left(\mathrm{s},[8 \mathrm{H}], \mathrm{OCH}_{2}\right), 3.78$ (br s, $[8 \mathrm{H}], \mathrm{OCH}_{2}$ ), 2.85 (v br s, $[8 \mathrm{H}]$, $\left.\mathrm{SCH}_{2}\right), 2.12$ (s, [6H], $\left.\mathrm{CH}_{3}, \mathrm{MeCN}\right) .{ }^{13} \mathrm{C}\left\{{ }^{1} \mathrm{H}\right\}$ NMR $\left(\mathrm{CD}_{2} \mathrm{Cl}_{2}\right.$, $298 \mathrm{~K}): \delta=162.31$ (q, $\left.J_{\mathrm{C}-\mathrm{B}}=49.9 \mathrm{~Hz}, \mathrm{BAr}^{\mathrm{F}} \mathrm{C} 1\right), 135.38\left(\mathrm{BAr}^{\mathrm{F}}\right.$ C2/6), 129.46 (qq, $\left.{ }^{2} J_{\mathrm{C}-\mathrm{F}}=31.6,2.9 \mathrm{~Hz}, \mathrm{BAr}^{\mathrm{F}} \mathrm{C} 3 / 5\right), 125.18$ (C, q, $\left.J_{\mathrm{C}-\mathrm{F}}=272 \mathrm{~Hz}, \mathrm{CF}_{3}\right), 119.50(\mathrm{C}, \mathrm{MeCN}), 118.10$ (CH, septet, $\left.{ }^{3} J_{\mathrm{C}-\mathrm{F}}=4.0 \mathrm{~Hz}, \mathrm{BAr}^{\mathrm{F}} \mathrm{C} 4\right), 71.38,70.16\left(\mathrm{OCH}_{2}\right), 31.50\left(\mathrm{SCH}_{2}\right)$, $2.28\left(\mathrm{CH}_{3}, \mathrm{MeCN}\right)$. IR (Nujol/ $\left.\mathrm{cm}^{-1}\right): 2303,2275$ (MeCN), 1612, 1282, 1148, 1090, 888, 682, 670, 580, 481, 450, 393.

$\left[\mathrm{Sr}\left([\mathbf{1 8}] \mathbf{a n e O}_{2} \mathbf{S}_{4}\right)(\mathbf{M e C N})_{2}\right]\left[\mathrm{BAr}^{\mathrm{F}}\right]_{2} \cdot \quad\left[\mathrm{Sr}(\mathrm{acacH})(\mathrm{MeCN})_{5}\right]\left[\mathrm{BAr}^{\mathrm{F}}\right]_{2}$ $(0.100 \mathrm{~g}, 0.047 \mathrm{mmol})$ and $[18] \mathrm{aneO}_{2} \mathrm{~S}_{4}(0.015 \mathrm{~g}, 0.047 \mathrm{mmol})$ afforded $0.085 \mathrm{~g}$ of a white solid in $86 \%$ yield. Anal. Required for $\mathrm{C}_{80} \mathrm{H}_{54} \mathrm{~B}_{2} \mathrm{~F}_{48} \mathrm{~N}_{2} \mathrm{O}_{2} \mathrm{~S}_{4} \mathrm{Sr}$ (2224.73): C, 43.19; H, 2.45; N, 1.26. Found: C, 43.25; H, 2.37; N, 1.18\%. ${ }^{1} \mathrm{H} \mathrm{NMR}\left(\mathrm{CD}_{2} \mathrm{Cl}_{2}, 298 \mathrm{~K}\right)$ : $\delta=7.73\left(\right.$ br s, [16H], BAr $\left.^{\mathrm{F}} \mathrm{H} 2 / 6\right), 7.58$ (s, [8H], $\left.\mathrm{BAr}^{\mathrm{F}} \mathrm{H} 4\right), 3.87$ $\left(\mathrm{t}, J=5.1 \mathrm{~Hz},[8 \mathrm{H}], \mathrm{OCH}_{2}\right), 2.95\left(\mathrm{~s},[8 \mathrm{H}], \mathrm{SCH}_{2}\right), 2.89(\mathrm{t}, J=$ $\left.5.2 \mathrm{~Hz},[8 \mathrm{H}], \mathrm{SCH}_{2}\right), 2.08$ (s, [6H], $\left.\mathrm{CH}_{3}, \mathrm{MeCN}\right) .{ }^{13} \mathrm{C}\left\{{ }^{1} \mathrm{H}\right\} \mathrm{NMR}$ $\left(\mathrm{CD}_{2} \mathrm{Cl}_{2}, 298 \mathrm{~K}\right): \delta=162.32\left({ }^{1} J_{\mathrm{C}-\mathrm{B}}=49.9 \mathrm{~Hz}, \mathrm{BAr}^{\mathrm{F}} \mathrm{C} 1\right), 135.39$ $\left(\mathrm{BAr}^{\mathrm{F}} \mathrm{C} 2 / 6\right), 129.43$ (qq, $\left.{ }^{2} J_{\mathrm{C}-\mathrm{F}}=31.6,2.9 \mathrm{~Hz}, \mathrm{BAr}^{\mathrm{F}} \mathrm{C} 3 / 5\right), 125.19$ $\left(\mathrm{q}, J_{\mathrm{C}-\mathrm{F}}=272 \mathrm{~Hz}, \mathrm{CF}_{3}\right), 119.24(\mathrm{C}, \mathrm{MeCN}), 118.11$ (septet, $\left.{ }^{3} J_{\mathrm{C}-\mathrm{F}}=4.0 \mathrm{~Hz}, \mathrm{BAr}^{\mathrm{F}} \mathrm{C} 4\right), 71.04\left(\mathrm{OCH}_{2}\right), 32.56,32.41\left(\mathrm{SCH}_{2}\right)$, $2.26\left(\mathrm{CH}_{3}, \mathrm{MeCN}\right)$. IR ( $\left.\mathrm{Nujol} / \mathrm{cm}^{-1}\right): 2308,2280$ (MeCN), 1611, 1281, 1127, 932, 899, 889, 839, 745, 683, 670, 383, 365.

$\left[\mathrm{Sr}\left([\mathbf{1 8}] \mathrm{aneO}_{4} \mathrm{Se}_{2}\right)\left(\mathrm{MeCN}_{2}\right]\left[\mathrm{BAr}^{\mathrm{F}}\right]_{2} \cdot\left[\mathrm{Sr}(\mathrm{acacH})(\mathrm{MeCN})_{5}\right]\left[\mathrm{BAr}^{\mathrm{F}}\right]_{2}\right.$ $(0.100 \mathrm{~g}, 0.047 \mathrm{mmol})$ and $[18] \mathrm{aneO}_{4} \mathrm{Se}_{2}(0.018 \mathrm{~g}, 0.047 \mathrm{mmol})$ afforded $0.077 \mathrm{~g}$ of a white solid in $72 \%$ yield. Anal. Required for $\mathrm{C}_{80} \mathrm{H}_{54} \mathrm{~B}_{2} \mathrm{~F}_{48} \mathrm{~N}_{2} \mathrm{O}_{4} \mathrm{Se}_{2} \mathrm{Sr}$ (2286.75): C, 42.02; H, 2.38; N, 1.22. Found: C, 42.14; H, 2.29; N, 1.25\%. ${ }^{1} \mathrm{H} \mathrm{NMR}\left(\mathrm{CD}_{2} \mathrm{Cl}_{2}, 298 \mathrm{~K}\right)$ : $\delta=7.73$ (br s, $\left.[16 \mathrm{H}], \mathrm{BAr}^{\mathrm{F}} \mathrm{H} 2 / 6\right), 7.58$ (s, [8H], BAr $\left.{ }^{\mathrm{F}} \mathrm{H} 4\right)$, 3.82-3.94 (m, [16H], $\mathrm{OCH}_{2}$ ), 2.87 (v br s, [8H], $\mathrm{SeCH}_{2}$ ), 2.15 (s, [6H] $\left., \mathrm{CH}_{3}, \mathrm{MeCN}\right) .{ }^{13} \mathrm{C}\left\{{ }^{1} \mathrm{H}\right\} \mathrm{NMR}\left(\mathrm{CD}_{2} \mathrm{Cl}_{2}, 298 \mathrm{~K}\right): \delta=162.33$ $\left(\mathrm{q},{ }^{1} J_{\mathrm{C}-\mathrm{B}}=49.9 \mathrm{~Hz}, \mathrm{BAr}^{\mathrm{F}} \mathrm{C} 1\right), 135.39\left(\mathrm{BAr}^{\mathrm{F}} \mathrm{C} 2 / 6\right), 129.46$ $\left(\mathrm{qq},{ }^{2} J_{\mathrm{C}-\mathrm{F}}=31.6,2.9 \mathrm{~Hz}, \mathrm{BAr}^{\mathrm{F}} \mathrm{C} 3 / 5\right), 125.20\left(\mathrm{q}, J_{\mathrm{C}-\mathrm{F}}=272 \mathrm{~Hz}\right.$, $\mathrm{CF}_{3}$ ), 120.20 (C, MeCN), 118.11 (septet, ${ }^{3} J_{\mathrm{C}-\mathrm{F}}=4.0 \mathrm{~Hz}$, BAr $\left.^{\mathrm{F}} \mathrm{C} 4\right), 71.43,71.33\left(\mathrm{OCH}_{2}\right), 25.24\left(\mathrm{SeCH}_{2}\right), 2.40\left(\mathrm{CH}_{3}\right.$, $\mathrm{MeCN})$. IR (Nujol/cm ${ }^{-1}$ ): 2305, 2275 (MeCN), 1611, 1284, 1140, 1117, 897, 886, 838, 682, 670, 579, 448, 392, 366.

$\left[\mathrm{Ba}\left([\mathbf{1 8}] \mathbf{a n e O}_{2} \mathbf{S}_{4}\right)(\mathbf{a c a c H})(\mathbf{M e C N})\right]\left[\mathrm{BAr}^{\mathbf{F}}\right]_{2} \cdot\left[\mathrm{Ba}(\mathrm{acacH})(\mathrm{MeCN})_{5}\right]$ $\left[\mathrm{BAr}^{\mathrm{F}}\right]_{2}(0.100 \mathrm{~g}, 0.046 \mathrm{mmol})$ and $[18] \mathrm{aneO}_{2} \mathrm{~S}_{4}(0.015 \mathrm{~g}$, $0.046 \mathrm{mmol}$ ) afforded $0.074 \mathrm{~g}$ of a white solid in $69 \%$ yield. Anal. Required for $\mathrm{C}_{83} \mathrm{H}_{59} \mathrm{~B}_{2} \mathrm{BaF}_{48} \mathrm{NO}_{4} \mathrm{~S}_{4}$ (2333.50): C, 42.72; $\mathrm{H}$, 2.55 ; N, 0.60. Found: C, 42.60; H, 2.63; N, 0.71\%. ${ }^{1} \mathrm{H}$ NMR $\left(\mathrm{CD}_{2} \mathrm{Cl}_{2}, 298 \mathrm{~K}\right): \delta=7.73\left(\mathrm{br} \mathrm{s},[16 \mathrm{H}], \mathrm{BAr}^{\mathrm{F}} \mathrm{H} 2 / 6\right), 7.58(\mathrm{~s},[8 \mathrm{H}]$, $\left.\mathrm{BAr}^{\mathrm{F}} \mathrm{H} 4\right), 3.86\left(\mathrm{t}, J=5.0 \mathrm{~Hz},[8 \mathrm{H}], \mathrm{OCH}_{2}\right), 3.84(\mathrm{~s},[2 \mathrm{H}]$, acacH $\left.\mathrm{CH}_{2}\right), 2.94\left(\mathrm{~s},[8 \mathrm{H}], \mathrm{SCH}_{2}\right), 2.86\left(\mathrm{t}, J=4.9 \mathrm{~Hz},[8 \mathrm{H}], \mathrm{SCH}_{2}\right), 2.29$ (s, [6H], acacH $\mathrm{CH}_{3}$ ), 2.05 (s, [6H], $\left.\mathrm{CH}_{3}, \mathrm{MeCN}\right) .{ }^{13} \mathrm{C}\left\{{ }^{1} \mathrm{H}\right\} \mathrm{NMR}$ $\left(\mathrm{CD}_{2} \mathrm{Cl}_{2}, 298 \mathrm{~K}\right): \delta=209.66(\mathrm{acacH} \mathrm{CO}), 162.34\left(\mathrm{q}, J_{\mathrm{C}-\mathrm{B}}=\right.$ $\left.49.9 \mathrm{~Hz}, \mathrm{BAr}^{\mathrm{F}} \mathrm{C} 1\right), 135.40\left(\mathrm{BAr}^{\mathrm{F}} \mathrm{C} 2 / 6\right), 129.48\left(\mathrm{qq},{ }^{2} J_{\mathrm{C}-\mathrm{F}}=31.6\right.$, 
$\left.2.9 \mathrm{~Hz}, \mathrm{BAr}^{\mathrm{F}} \mathrm{C} 3 / 5\right), 125.21\left(\mathrm{q}, J_{\mathrm{C}-\mathrm{F}}=272 \mathrm{~Hz}, \mathrm{CF}_{3}\right), 118.12$ (septet, $\left.{ }^{3} J_{\mathrm{C}-\mathrm{F}}=4.0 \mathrm{~Hz}, \mathrm{BAr}{ }^{\mathrm{F}} \mathrm{C} 4\right), 71.11\left(\mathrm{OCH}_{2}\right), 53.63\left(\mathrm{CH}_{2}\right.$, acacH), $33.05\left(\mathrm{CH}_{3}, \mathrm{acacH}\right), 32.89,32.61\left(\mathrm{SCH}_{2}\right), 2.24\left(\mathrm{CH}_{3}\right.$, MeCN). IR (Nujol/cm ${ }^{-1}$ ): 2306, 2268 (MeCN), 1737 (acacH), 1611, 1283, 1117, 937, 887, 839, 683, 670.

$\left[\mathrm{Ba}\left([18] \mathrm{aneO}_{4} \mathrm{Se}_{2}\right)(\mathbf{a c a c H})(\mathrm{MeCN})\right]\left[\mathrm{BAr}^{\mathrm{F}}\right]_{2} \cdot\left[\mathrm{Ba}(\mathrm{acacH})(\mathrm{MeCN})_{5}\right]-$ $\left[\mathrm{BAr}^{\mathrm{F}}\right]_{2}(0.100 \mathrm{~g}, 0.046 \mathrm{mmol})$ and $[18] \mathrm{aneO}_{4} \mathrm{Se}_{2}(0.018 \mathrm{~g}$, $0.046 \mathrm{mmol}$ ) afforded $0.086 \mathrm{~g}$ of a white solid in $78 \%$ yield. Anal. Required for $\mathrm{C}_{83} \mathrm{H}_{59} \mathrm{~B}_{2} \mathrm{BaF}_{48} \mathrm{NO}_{6} \mathrm{Se}_{2}$ (2409.52): C, 41.62; $\mathrm{H}, 2.48$; N, 0.58. Found: C, 41.51; H, 2.35; N, 0.63\%. ${ }^{1} \mathrm{H}$ NMR $\left(\mathrm{CD}_{2} \mathrm{Cl}_{2}, 298 \mathrm{~K}\right): \delta=7.72$ (br s, [16H], $\left.\mathrm{BAr}^{\mathrm{F}} \mathrm{H} 2 / 6\right), 7.58(\mathrm{~s},[8 \mathrm{H}]$, $\left.\mathrm{BAr}^{\mathrm{F}} \mathrm{H} 4\right), 3.88\left(\mathrm{t}, J=5.4 \mathrm{~Hz},[8 \mathrm{H}], \mathrm{OCH}_{2}\right), 3.85(\mathrm{~s},[2 \mathrm{H}], \mathrm{acacH}$ $\mathrm{CH}_{2}$ ), $3.80\left(\mathrm{~s},[8 \mathrm{H}], \mathrm{OCH}_{2}\right), 2.89\left(\mathrm{t}, J=5.4 \mathrm{~Hz},[8 \mathrm{H}], \mathrm{SeCH}_{2}\right.$ ), 2.30 (s, [6H], $\left.\mathrm{CH}_{3}, \mathrm{acacH}\right), 2.06$ (s, [3H], $\left.\mathrm{CH}_{3}, \mathrm{MeCN}\right)$. ${ }^{13} \mathrm{C}\left\{{ }^{1} \mathrm{H}\right\} \operatorname{NMR}\left(\mathrm{CD}_{2} \mathrm{Cl}_{2}, 298 \mathrm{~K}\right): \delta=209.76$ (acacH CO), 162.32 $\left(\mathrm{q},{ }^{1} J_{\mathrm{C}-\mathrm{B}}=49.9 \mathrm{~Hz}, \mathrm{BAr}^{\mathrm{F}} \mathrm{C} 1\right), 135.39\left(\mathrm{BAr}^{\mathrm{F}} \mathrm{C} 2 / 6\right), 129.47$ (qq, $\left.{ }^{2} J_{\mathrm{C}-\mathrm{F}}=31.6,2.9 \mathrm{~Hz}, \mathrm{BAr}^{\mathrm{F}} \mathrm{C} 3 / 5\right), 125.19\left(\mathrm{q}, J_{\mathrm{C}-\mathrm{F}}=272 \mathrm{~Hz}, \mathrm{CF}_{3}\right.$ ), $120.06(\mathrm{C}, \mathrm{MeCN}), 118.10$ (septet, ${ }^{3} J_{\mathrm{C}-\mathrm{F}}=4.0 \mathrm{~Hz}, \mathrm{BAr}^{\mathrm{F}} \mathrm{C} 4$ ), 71.47, $71.08\left(\mathrm{OCH}_{2}\right), 53.53\left(\mathrm{CH}_{2}, \mathrm{acacH}\right), 33.07\left(\mathrm{CH}_{3}, \mathrm{acacH}\right)$, $25.43\left(\mathrm{SeCH}_{2}\right), 2.27\left(\mathrm{CH}_{3}, \mathrm{MeCN}\right) . \mathrm{IR}\left(\mathrm{Nujol} / \mathrm{cm}^{-1}\right): 2299,2266$ (MeCN), 1740 (acacH), 1611, 1283, 1116, 1007, 885, 839, 744, $682,669448,366$.

$\left[\mathrm{Ca}\left([18]\right.\right.$ aneO $\left._{4} \mathrm{~S}_{2}\right)\left(2,2^{\prime}\right.$-bipyridyl $\left.)\right]\left[\mathrm{BAr}^{\mathrm{F}}\right]_{2}$. $\left[\mathrm{Ca}\left([18] \mathrm{aneO}_{4} \mathrm{~S}_{2}\right)-\right.$ $\left.(\mathrm{MeCN})_{2}\right]\left[\mathrm{BAr}^{\mathrm{F}}\right]_{2} \quad(0.030 \mathrm{~g}, 0.014 \mathrm{mmol})$ was dissolved in $\mathrm{CH}_{2} \mathrm{Cl}_{2}(10 \mathrm{~mL})$ and a solution of 2,2'-bipyridyl $(0.002 \mathrm{~g}$, $0.014 \mathrm{mmol})$ in $\mathrm{CH}_{2} \mathrm{Cl}_{2}(5 \mathrm{~mL})$ was added. The reaction was stirred for $16 \mathrm{~h}$, then concentrated to $\sim 5 \mathrm{~mL}$ and hexane was layered on top. Colourless crystals were produced which were isolated by decanting away the solvent and drying in vacuo. Yield: $0.031 \mathrm{~g}$, 99\%. Anal. Required for $\mathrm{C}_{86} \mathrm{H}_{56} \mathrm{~N}_{2} \mathrm{O}_{4} \mathrm{~B}_{2} \mathrm{CaF}_{48} \mathrm{~S}_{2}$ (2219.31): C, 46.54; H, 2.54; N, 1.26. Found: C, 46.39; H, 2.41; $\mathrm{N}, 1.35 .{ }^{1} \mathrm{H}$ NMR $\left(\mathrm{CD}_{2} \mathrm{Cl}_{2}, 298 \mathrm{~K}\right): \delta=7.84-8.59$ (br m, [8H], bipy), 7.73 (br s, [16H], BAr ${ }^{\mathrm{F}} \mathrm{H} 2 / 6$ ), 7.57 (s, [8H], BAr $\left.{ }^{\mathrm{F}} \mathrm{H} 4\right), 4.08$ (vbr s, $[8 \mathrm{H}], \mathrm{OCH}_{2}$ ), 3.94 (br s, $\left.[8 \mathrm{H}], \mathrm{OCH}_{2}\right), 2.77$ (vbr s, $[8 \mathrm{H}]$, $\left.\mathrm{SCH}_{2}\right) \cdot{ }^{13} \mathrm{C}\left\{{ }^{1} \mathrm{H}\right\}$ NMR $\left(\mathrm{CD}_{2} \mathrm{Cl}_{2}, 298 \mathrm{~K}\right): \delta=162.34\left(\mathrm{q},{ }^{1} J_{\mathrm{C}-\mathrm{B}}=\right.$ $\left.50.0 \mathrm{~Hz}, \mathrm{BAr}^{\mathrm{F}} \mathrm{C} 1\right), 135.40\left(\mathrm{BAr}^{\mathrm{F}} \mathrm{C} 2 / 6\right), 129.48\left(\mathrm{qq},{ }^{2} J_{\mathrm{C}-\mathrm{F}}=31.6\right.$, $\left.2.9 \mathrm{~Hz}, \mathrm{BAr}^{\mathrm{F}} \mathrm{C} 3 / 5\right), 125.20$ (q, $\left.{ }^{1} J_{\mathrm{C}-\mathrm{F}}=272 \mathrm{~Hz}, \mathrm{CF}_{3}\right), 118.01$ (septet, $\left.{ }^{3} J_{\mathrm{C}-\mathrm{F}}=4.0 \mathrm{~Hz}, \mathrm{BAr}{ }^{\mathrm{F}} \mathrm{C} 4\right), 69.54,68.20\left(\mathrm{OCH}_{2}\right), 31.75\left(\mathrm{SCH}_{2}\right)$.

\section{Results and discussion}

\section{Metal synthons}

We initially attempted to prepare the MeCN complexes $\left[\mathrm{M}(\mathrm{MeCN})_{x}\right]\left[\mathrm{BAr}^{\mathrm{F}}\right]_{2}(\mathrm{M}=\mathrm{Mg}, x=6 ; \mathrm{M}=\mathrm{Ca}, \mathrm{Sr}$ or $\mathrm{Ba}, x=8)$ by salt metathesis between the appropriate $\mathrm{MI}_{2}$ and $\mathrm{Na}\left[\mathrm{BAr}{ }^{\mathrm{F}}\right]$ in anhydrous MeCN solution (Scheme 1), since it was anticipated

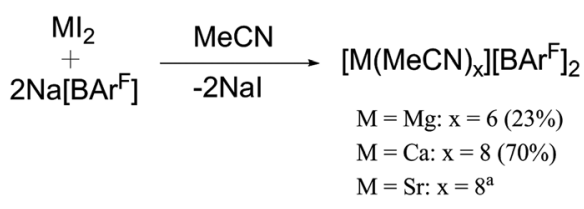

Scheme 1 Synthesis of $\left[\mathrm{M}(\mathrm{MeCN})_{x}\right]\left[\mathrm{BAr}^{\mathrm{F}}\right]_{2}(\mathrm{M}=\mathrm{Mg}, \mathrm{Ca}, \mathrm{Sr})$. ${ }^{\text {a Product }}$ contaminated with $\mathrm{Na}\left[\mathrm{BAr}{ }^{\mathrm{F}}\right]$. that these compounds would serve as more readily soluble synthons for accessing soft donor macrocyclic complexes for the Group 2 cations, while incorporating weakly coordinating $\left[\mathrm{BAr}^{\mathrm{F}}\right]^{-}$anions. This was successful for $\mathrm{M}=\mathrm{Mg}$ and $\mathrm{Ca}$, yielding $\left[\mathrm{Mg}(\mathrm{MeCN})_{6}\right]\left[\mathrm{BAr}^{\mathrm{F}}\right]_{2}$ and $\left[\mathrm{Ca}(\mathrm{MeCN})_{8}\right]\left[\mathrm{BAr}^{\mathrm{F}}\right]_{2}$ after purification as described in the Experimental section. However, for $\mathbf{M}=\mathrm{Sr}$, salt metathesis resulted in products which retained a large amount of $\mathrm{Na}\left[\mathrm{BAr}^{\mathrm{F}}\right]$, and although a small number of crystals formed in one reaction which were identified as $\left[\mathrm{Sr}(\mathrm{MeCN})_{8}\right]\left[\mathrm{BAr}^{\mathrm{F}}\right]_{2}$ (see below), this complex could not be obtained in sufficient yield or purity to be a useful synthon. The salt metathesis also failed in the barium case. As an alternative route, the reaction of $\left[\mathrm{M}(\mathrm{acac})_{2}\right](\mathrm{M}=\mathrm{Sr}$ or $\mathrm{Ba}$, acacH $=$ acetylacetone) with $\left[\mathrm{H}\left(\mathrm{OEt}_{2}\right)_{2}\right]\left[\mathrm{BAr}^{\mathrm{F}}\right]^{17}$ in anhydrous MeCN was used to prepare $\left[\mathrm{M}(\mathrm{MeCN})_{5}(\mathrm{acacH})\right]\left[\mathrm{BAr}^{\mathrm{F}}\right]_{2}, \quad(\mathrm{M}=\mathrm{Sr}$ or $\mathrm{Ba})$ which proved to be readily isolated (Scheme 2 ). These complexes were characterised by microanalysis, ${ }^{1} \mathrm{H}$ and ${ }^{13} \mathrm{C}\left\{{ }^{1} \mathrm{H}\right\}$ NMR spectroscopy. Their IR spectra are consistent with coordination of neutral acacH. ${ }^{22}$ Colourless, moisture sensitive crystals of $[\mathrm{Mg}$ $\left.(\mathrm{MeCN})_{6}\right]\left[\mathrm{BAr}^{\mathrm{F}}\right]_{2}$ were grown from $\mathrm{CH}_{2} \mathrm{Cl}_{2} / n$-hexane, and the $\mathrm{X}$-ray crystal structure showed the expected regular octahedral cation (Fig. 1). The cation has been structurally characterised in several salts including $\left[\mathrm{Mg}(\mathrm{MeCN})_{6}\right]\left[\mathrm{MgBr}_{4}\right]^{7}$ and $[\mathrm{Mg}$ $\left.(\mathrm{MeCN})_{6}\right]\left[\mathrm{AlCl}_{4}\right]_{2},{ }^{23}$ and the dimensions are comparable: the present structure serves to confirm the identity of the reagent.

The ${ }^{1} \mathrm{H}$ and ${ }^{13} \mathrm{C}\left\{{ }^{1} \mathrm{H}\right\}$ NMR spectra show the $\left[\mathrm{BAr}^{\mathrm{F}}\right]^{-}$anions, with resonances due to the $\mathrm{CH}_{3}$ group of MeCN at $\delta=2.16$ and $\delta=2.18$ respectively, shifted from the liquid resonances at

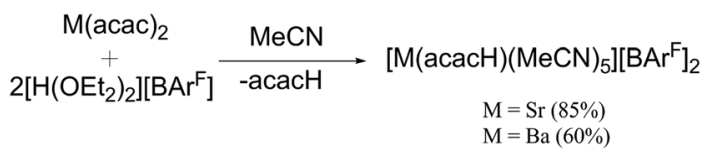

Scheme 2 Synthesis of $\left[\mathrm{M}(\mathrm{acacH})(\mathrm{MeCN})_{5}\right]\left[\mathrm{BAr}{ }^{\mathrm{F}}\right]_{2}(\mathrm{M}=\mathrm{Sr}, \mathrm{Ba})$.

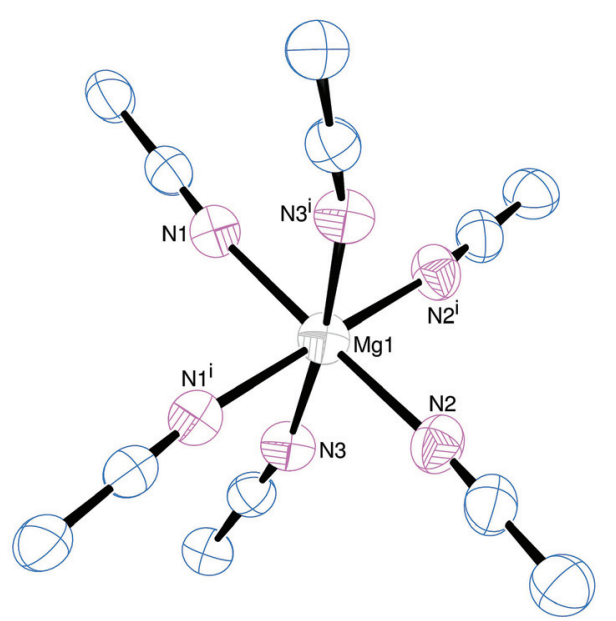

Fig. 1 ORTEP representation of the dication of $\left[\mathrm{Mg}(\mathrm{MeCN})_{6}\right]\left[\mathrm{BAr}{ }^{\mathrm{F}}\right]_{2}$. Ellipsoids are shown at the $50 \%$ probability level and the hydrogen atoms are omitted for clarity. Selected bond lengths $(\AA)$ : $M g-N 1=2.165$ (5), Mg-N2 = 2.143(5), Mg-N3 = 2.135(4). Symmetry code: (i) $-x,-y, z$. 


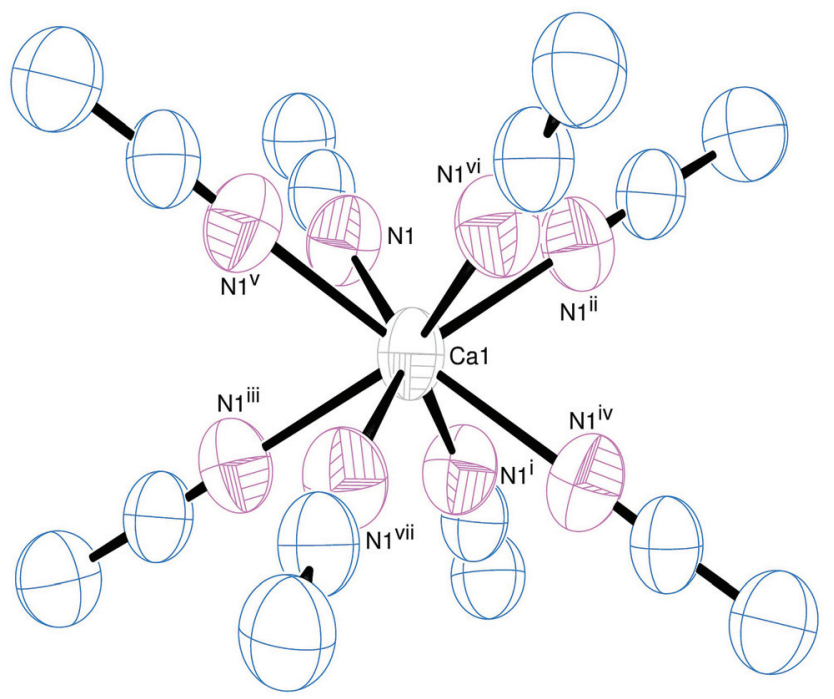

Fig. 2 ORTEP representation of the dication $\left[\mathrm{Ca}(\mathrm{MeCN})_{8}\right]^{2+}$. Ellipsoids are shown at the $50 \%$ probability level and hydrogen atoms omitted for clarity. Bond lengths ( $(\AA)$ : $\mathrm{Ca}-\mathrm{N}=2.514(5), \mathrm{N}-\mathrm{C}=1.136(7)$. Symmetry codes: (i) $x, 0.5-y, 0.5-z$; (ii) $0.5-x, y, z$; (iii) $0.5-x, 0.5-y, 0.5-z$; (iv) $x, 0.5-y, z$; (v) $0.5-x, y, 0.5-z$; (vi) $0.5-x, 0.5-y, z$; (vii) $x, y, 0.5-z$.

$\delta=1.96$ and $\delta=1.79$. The IR spectrum (liquid film) shows two resonances at 2252 and $2293 \mathrm{~cm}^{-1}$ assigned as $\nu(\mathrm{CN})$ and $\nu(\mathrm{C}-\mathrm{C})+\delta\left(\mathrm{CH}_{3}\right)$ of similar intensity due to Fermi resonance. ${ }^{24}$ In $\left[\mathrm{Mg}(\mathrm{MeCN})_{6}\right]\left[\mathrm{BAr}^{\mathrm{F}}\right]_{2}$ both bands are found at higher frequency $\left(2295,2321 \mathrm{~cm}^{-1}\right)$, consistent with coordination to the metal. Similar patterns were observed for all the complexes in this work, confirming the MeCN is coordinated in all cases.

Extremely moisture sensitive crystals of $\left[\mathrm{Ca}(\mathrm{MeCN})_{8}\right]\left[\mathrm{BAr}^{\mathrm{F}}\right]_{2}$ were grown from $\mathrm{CH}_{2} \mathrm{Cl}_{2} / n$-hexane, but these partially lose MeCN on drying with both microanalysis and integration of the ${ }^{1} \mathrm{H}$ NMR spectrum showing $\sim 6 \mathrm{MeCN} / \mathrm{Ca}$ in the vacuumdried product. ${ }^{25}$ The structure (Fig. 2) shows a square antiprismatic cation with no interaction with the $\left[\mathrm{BAr}^{\mathrm{F}}\right]^{-}$anions. This cation has not been reported previously, although the $\mathrm{Ca}-\mathrm{NCMe}$ bond lengths are similar to those in other calcium complexes including $\left[\mathrm{Ca}(18 \text {-crown-6)( } \mathrm{MeCN})_{2}\right]\left[\mathrm{SbCl}_{6}\right]_{2},{ }^{13}$ $\left[\mathrm{Ca}(18 \text {-crown-6)(MeCN })_{2}\right]\left[\mathrm{BPh}_{4}\right]_{2}{ }^{26}$ and $\left[\mathrm{Ca}(\mathrm{MeCN})_{5} \mathrm{I}_{2}\right]^{7}$

The spectroscopic properties of $\left[\mathrm{Sr}(\mathrm{MeCN})_{8}\right]\left[\mathrm{BAr}^{\mathrm{F}}\right]_{2}$ are similar to those of the calcium analogue. A few modest quality crystals of this complex were formed as a by-product from the salt metathesis reaction and also deposited from an MeCN solution of $\left[\mathrm{Sr}(\mathrm{MeCN})_{5}(\mathrm{acacH})\right]\left[\mathrm{BAr}^{\mathrm{F}}\right]_{2}$. The X-ray structure showed them to contain a square antiprismatic cation (see Fig. S1, ESI $\dagger$ ).

The $\left[\mathrm{M}(\mathrm{acacH})(\mathrm{MeCN})_{5}\right]\left[\mathrm{BAr}^{\mathrm{F}}\right]_{2}(\mathrm{M}=\mathrm{Sr}, \mathrm{Ba})$ proved to be useful synthons for the heterocrown complexes. The IR spectra show coordinated MeCN and acacH, and although X-ray quality crystals were not obtained, they probably contain seven-coordinate metal centres.

\section{Heterocrown complexes}

The reaction of $\left[\mathrm{Ca}(\mathrm{MeCN})_{8}\right]\left[\mathrm{BAr}^{\mathrm{F}}\right]_{2}$ with $[18] \mathrm{aneO}_{2} \mathrm{~S}_{4}$, [18] aneO $_{4} \mathrm{~S}_{2}$, [18] $\mathrm{aneO}_{4} \mathrm{Se}_{2}$ and 18-crown-6 afforded complexes
$\left[\mathrm{Ca}(\right.$ crown $\left.)(\mathrm{MeCN})_{2}\right]\left[\mathrm{BAr}^{\mathrm{F}}\right]_{2}$. In all cases the ${ }^{1} \mathrm{H}$ and ${ }^{13} \mathrm{C}\left\{{ }^{1} \mathrm{H}\right\}$ NMR spectra show small high frequency shifts for the $\mathrm{CH}_{2} \mathrm{O}, \mathrm{CH}_{2} \mathrm{~S} / \mathrm{Se}$ and $\mathrm{CH}_{3} \mathrm{CN}$ groups consistent with coordination. The IR spectra are dominated by the $\left[\mathrm{BAr}^{\mathrm{F}}\right]^{-}$anion vibrations, but show small high frequency shifts in the vibrations due to MeCN. X-ray quality crystals were grown for the adducts with [18] $\mathrm{aneO}_{2} \mathrm{~S}_{4},[18] \mathrm{aneO}_{4} \mathrm{Se}_{2}$ (Fig. 3) and 18-crown-6 (Fig. S2, ESI $\dagger$ ).

For each complex an eight-coordinate $\mathrm{Ca}^{2+}$ centre sitting in the middle of a hexadentate macrocycle with trans MeCN ligands was observed. Similar structures have been reported for $\left[\mathrm{Ca}\left(18\right.\right.$-crown-6) $\left.\mathrm{I}_{2}\right],{ }^{27}\left[\mathrm{Ca}(18 \text {-crown-6)(hmpa) })_{2}\right]^{2+}(\mathrm{hmpa}=$ hexamethylphosphoramide $^{26}$ and [Ca(18-crown-6)(MeCN) $\left.)_{2}\right]$ $\left[\mathrm{SbCl}_{6}\right]_{2},{ }^{13}$ although in contrast $\left[\mathrm{Ca}\right.$ (heterocrown) $\left.\mathrm{I}_{2}\right]$ (heterocrown $=[18] \mathrm{aneO}_{4} \mathrm{Se}_{2}$ or $\left.[18] \mathrm{aneO}_{2} \mathrm{~S}_{4}\right),{ }^{13} \quad[\mathrm{Ca}(18$-crown-6)$\left.\left(\mathrm{O}_{3} \mathrm{SCF}_{3}\right)_{2}\right]^{13}$ and $\left[\mathrm{Ca}\left([18] \mathrm{aneO}_{4} \mathrm{~S}_{2}\right)\left(\mathrm{OH}_{2}\right)_{2}\right] \mathrm{I}_{2}{ }^{13}$ contain puckered macrocycles with cis coordinated monodentate ligands. It is probable that the energy difference between the different arrangements is small and the cation geometry may be influenced by intermolecular interactions and crystal packing. Eight coordination, either dodecahedral or square antiprismatic, is relatively common for the heavier alkaline earth metals, ${ }^{2,4}$ and whilst the geometry is readily established for those containing eight monodentate ligands, ${ }^{28}$ the distortions resulting with polydentates can make identifying the coordination sphere difficult. Lippard and Russ ${ }^{29}$ detailed a method for mathematically distinguishing between the two polyhedra, which required the identification of the plane of best fit which encompassed the metal centre and four of the eight coordinating atoms, then calculating the dihedral angle between this plane and the plane defined by the metal centre and the remaining four coordinating atoms. For an ideal dodecahedron this angle will be $90^{\circ}$ whereas for an ideal square antiprism this angle will be $77.4^{\circ}$. A " $\tau_{8}$ " parameter can be defined as $(\theta-77.4) / 12.6$ where $\theta$ is the dihedral angle between the two planes of best fit. A $\tau_{8}$ value of 0 would correspond to an ideal square antiprismatic geometry and a $\tau_{8}$ value of 1 would correspond to an ideal dodecahedral geometry, with intermediate values indicating the degree of distortion away from the two ideals.

For the [18]ane $\mathrm{O}_{2} \mathrm{~S}_{4}$ complex the $\tau_{8}$ parameter is calculated to be 0.29 and for the [18] $\mathrm{aneO}_{4} \mathrm{Se}_{2}$ complex the $\tau_{8}$ parameter is 0.93 . This indicates that the latter complex is very close to an ideal dodecahedral geometry, whereas the former is better described as square antiprismatic, but with a high degree of distortion (Fig. 3(c) and (d)). This contrasts markedly with the $\left[\mathrm{Ca}\right.$ (heterocrown) $\left.\mathrm{I}_{2}\right]$ complexes where the macrocycle conformation was significantly folded because the iodide ligands bound in a cis manner and the geometry of the complexes was close to square antiprismatic. The $\left[\mathrm{Ca}(18 \text {-crown-6)(MeCN })_{2}\right]^{2+}$ dication (Fig. S2, ESI $\dagger$ ) has a $\tau_{8}$ value of 0.90 indicating that it is close to a dodecahedral geometry.

Changes in the macrocyclic coordination geometry do not significantly affect the $\mathrm{Ca}-\mathrm{S}$ and $\mathrm{Ca}-\mathrm{Se}$ bond lengths which are very similar between the iodide $\mathrm{e}^{13}$ and MeCN complexes, although there are some slight differences in the $\mathrm{Ca}-\mathrm{O}$ bond 


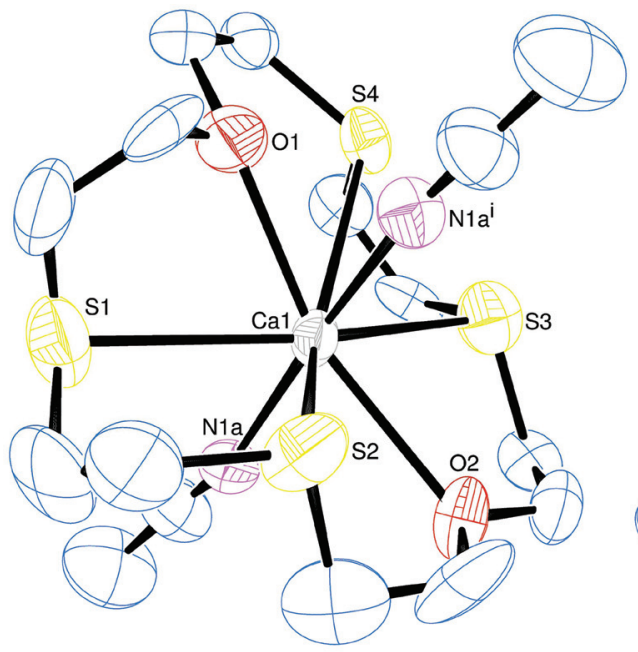

(a)

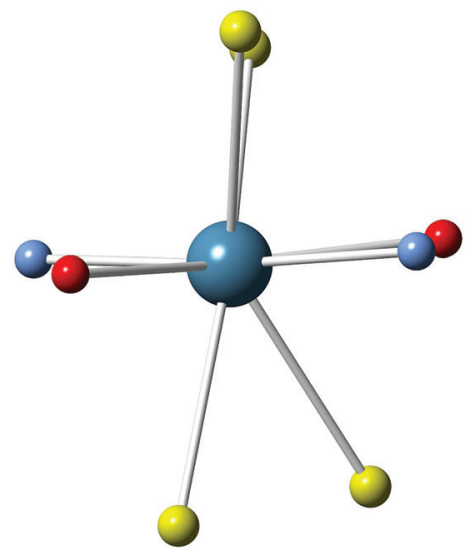

(c)

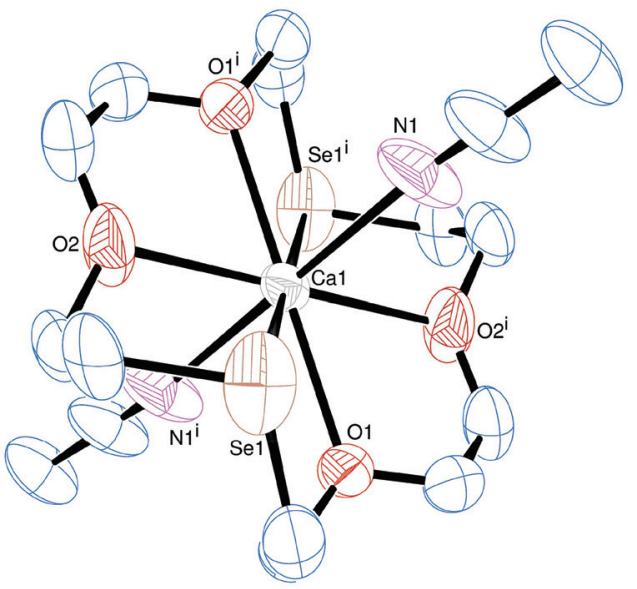

(b)

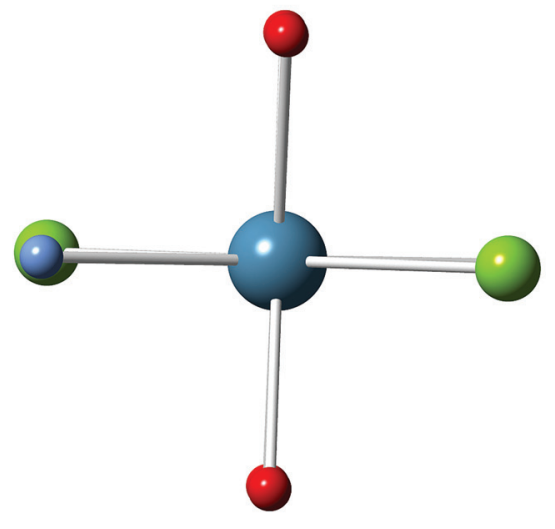

(d)

Fig. 3 (a) the cation of $\left[\mathrm{Ca}\left([18] \mathrm{aneO}{ }_{2} \mathrm{~S}_{4}\right)(\mathrm{MeCN})_{2}\right][\mathrm{BAr}]_{2}$ with the positional disorder and hydrogen atoms omitted for clarity. Selected bond lengths $(\AA)$ and angles $\left({ }^{\circ}\right): \mathrm{Ca}-\mathrm{O} 1=2.52(1), \mathrm{Ca}-\mathrm{O} 2=2.668(8), \mathrm{Ca}-\mathrm{S} 1=2.986(4), \mathrm{Ca}-\mathrm{S} 2=2.954(2), \mathrm{Ca}-\mathrm{S} 3=2.906(4), \mathrm{Ca}-\mathrm{S} 4=2.962(3), \mathrm{Ca}-\mathrm{N} 1 \mathrm{a}=2.37(1)$; $\mathrm{O} 1-\mathrm{Ca}-\mathrm{S} 1=65.3(2), \mathrm{S} 1-\mathrm{Ca}-\mathrm{S} 2=73.1(1), \mathrm{S} 2-\mathrm{Ca}-\mathrm{O} 2=68.3(2) \mathrm{O} 2-\mathrm{Ca}-\mathrm{S} 3=70.1(2), \mathrm{S} 3-\mathrm{Ca}-\mathrm{S} 4=54.2(1), \mathrm{S} 4-\mathrm{Ca}-\mathrm{O} 1=59.2(2), \mathrm{S} 1-\mathrm{Ca}-\mathrm{S} 3=153.8$ (1), $\mathrm{S} 2-\mathrm{Ca}-\mathrm{S} 4=146.0(1), \mathrm{O} 1-\mathrm{Ca}-\mathrm{O} 2=154.3(3)$. (b) The cation of $\left[\mathrm{Ca}\left([18]\right.\right.$ ane $\left.{ }_{4} \mathrm{Se}_{2}\right)\left(\mathrm{MeCN}_{2}\right][\mathrm{BAr}]_{2}$ with hydrogens and $\left[\mathrm{BAr} \mathrm{F}^{-}\right.$anions omitted for clarity. Selected bond lengths $(\AA)$ and angles $\left({ }^{\circ}\right): \mathrm{Ca}-\mathrm{O} 1=2.461(4), \mathrm{Ca}-\mathrm{O} 2=2.455(5), \mathrm{Ca}-\mathrm{Se} 1=3.1526(9), \mathrm{Ca}-\mathrm{N} 1=2.488(5) ; \mathrm{O} 1-\mathrm{Ca}-\mathrm{Se} 1=67.0(1)$, $\mathrm{O} 2-\mathrm{Ca}-\mathrm{Se} 1=66.0(1), \mathrm{O} 1-\mathrm{Ca}-\mathrm{O} 2^{i}=64.7(1)$. Symmetry code: (i) $1-x, 1-y,-z$. View of the metal coordination sphere for $\left[\mathrm{Ca}\left([18] \mathrm{anO} \mathrm{O}_{2} \mathrm{~S}_{4}\right)\right]^{2+}$ (c) and $\left[\mathrm{Ca}\left([18] \mathrm{aneO}_{4} \mathrm{Se}_{2}\right)\right]^{2+}$ (d) illustrating the differences from the (near) dodecahedral geometry present in the latter (colour key: blue $=\mathrm{N}$, red $=\mathrm{O}$, yellow $=\mathrm{S}$, green $=\mathrm{Se}$ )

lengths. Additionally, a slight shortening of the $\mathrm{Ca}-\mathrm{NCCH}_{3}$ bond lengths is observed for the macrocyclic complexes compared to $\left[\mathrm{Ca}(\mathrm{MeCN})_{8}\right]\left[\mathrm{BAr}^{\mathrm{F}}\right]_{2}$, although it should be noted that there was a significant amount of positional disorder present in the $[18] \mathrm{aneO}_{2} \mathrm{~S}_{4}$ complex (including the MeCN ligands) and care is needed in making the comparisons.

The MeCN ligands can be displaced by neutral donors, for example 2,2'-bipyridyl, which formed $\left[\mathrm{Ca}\left([18] \mathrm{aneO}_{4} \mathrm{~S}_{2}\right)(\right.$ bipy)]$\left[\mathrm{BAr}{ }^{\mathrm{F}}\right]_{2}$. The ${ }^{1} \mathrm{H}$ and ${ }^{13} \mathrm{C}\left\{{ }^{1} \mathrm{H}\right\}$ NMR spectra contained broad resonances indicative of a dissociating system in $\mathrm{CD}_{2} \mathrm{Cl}_{2}$ solution at ambient temperatures, but the identity was confirmed by the X-ray crystal structure (Fig. 4).
In order to accommodate the bidentate bipy ligand the [18] $\mathrm{aneO}_{4} \mathrm{~S}_{2}$ coordination becomes puckered, closer to that previously observed for the $\left[\mathrm{Ca}\left(18-\mathrm{aneO}_{4} \mathrm{~S}_{2}\right) \mathrm{I}_{2}\right]$ where the iodide ligands were cis and the geometry is close to dodecahedral, $\tau_{8}=0.82$.

Heterocrown complexes of strontium were obtained through reaction of $\left[\mathrm{Sr}(\mathrm{acacH})(\mathrm{MeCN})_{5}\right]\left[\mathrm{BAr}^{\mathrm{F}}\right]_{2}$ with $[18] \mathrm{aneO}_{4} \mathrm{~S}_{2}$, [18] $\mathrm{aneO}_{2} \mathrm{~S}_{4}$ and [18] $\mathrm{aneO}_{4} \mathrm{Se}_{2}$, forming [ $\mathrm{Sr}\left(\right.$ heterocrown) $(\mathrm{MeCN})_{2}$ ]$\left[\mathrm{BAr}^{\mathrm{F}}\right]_{2}$. Notably the acacH ligand is lost but two MeCN ligands were retained, as shown by the ${ }^{1} \mathrm{H}$ and ${ }^{13} \mathrm{C}\left\{{ }^{1} \mathrm{H}\right\}$ NMR data. This was confirmed by the X-ray structures of $\left[\operatorname{Sr}\left([18] \mathrm{aneO}_{4} \mathrm{~S}_{2}\right)\right.$ $\left.(\mathrm{MeCN})_{2}\right]\left[\mathrm{BAr}^{\mathrm{F}}\right]_{2}$ and $\left[\mathrm{Sr}\left([18] \mathrm{aneO}_{4} \mathrm{Se}_{2}\right)\left(\mathrm{MeCN}_{2}\right]\left[\mathrm{BAr}^{\mathrm{F}}\right]_{2}\right.$ (Fig. 5). 


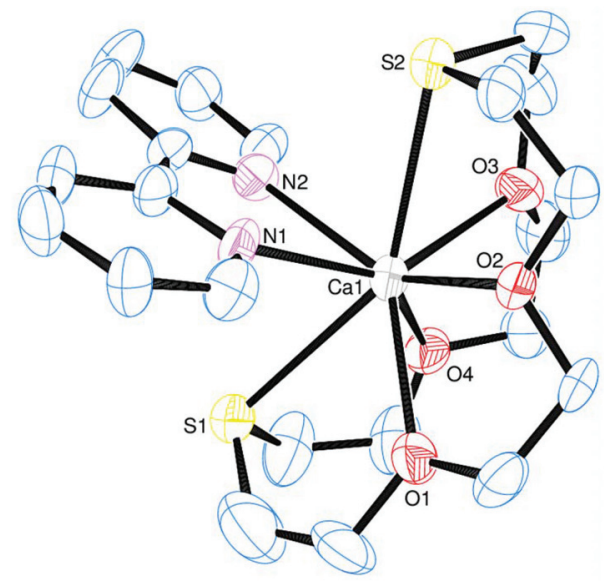

Fig. 4 The cation of $\left[\mathrm{Ca}\left([18] a n e \mathrm{O}_{4} \mathrm{~S}_{2}\right)(\right.$ bipy $\left.)\right]\left[\mathrm{BAr}{ }^{\mathrm{F}}\right]_{2}$. Ellipsoids are shown at the $50 \%$ probability level and the hydrogen atoms omitted for clarity. Selected bond lengths $(\AA)$ and angles $\left({ }^{\circ}\right)$ : $\mathrm{Ca}-\mathrm{O} 1=2.497(6), \mathrm{Ca}-\mathrm{O} 2=$ $2.433(6), \mathrm{Ca}-\mathrm{O} 3=2.514(6) \mathrm{Ca}-\mathrm{O} 4=2.462(6), \mathrm{Ca}-\mathrm{S} 1=2.943(4), \mathrm{Ca}-\mathrm{S} 2$ $=2.935(4), \mathrm{Ca}-\mathrm{N} 1=2.461(7), \mathrm{Ca}-\mathrm{N} 2=2.458(7) ; \mathrm{S} 1-\mathrm{Ca}-\mathrm{S} 2$ = 145.23(9), $\mathrm{S} 1-\mathrm{Ca}-\mathrm{O} 1=67.7(2), \mathrm{O} 1-\mathrm{Ca}-\mathrm{O} 4=87.0(2)$.

The structures revealed that both complexes adopted 8-coordinate geometries with a hexadentate macrocycle and two trans MeCN ligands. The $\tau_{8}$ parameter for the [18] $\operatorname{aneO}_{4} \mathrm{~S}_{2}$ complex was 0.73 and for the $[18] \mathrm{aneO}_{4} \mathrm{Se}_{2}$ complex it was 0.89 indicating that distorted dodecahedral is the best description, albeit severely distorted for the former. The $\mathrm{Sr}-\mathrm{S}$ and $\mathrm{Sr}-\mathrm{Se}$ bond lengths are very similar to those reported for the 9-coordinate $\left[\mathrm{Sr}(\right.$ macrocycle $\left.)\left(\mathrm{OH}_{2}\right)_{3}\right] \mathrm{I}_{2}$ complexes, ${ }^{13}$ although the $\mathrm{Sr}-\mathrm{O}$ bond lengths are slightly shorter than those previously reported which may be a result of lower coordination number
(8 vs. 9). No evidence for any $\mathrm{Sr} \cdots\left[\mathrm{BAr}^{\mathrm{F}}\right]$ interactions was observed, although such interactions were found with the larger alkali metals. ${ }^{11}$

The only example of an oxathia-crown complex of barium is in the polymer $\left[\mathrm{Ba}\left\{\mathrm{Cu}(\mathrm{SCN})_{3}\left([18] \mathrm{aneO}_{4} \mathrm{~S}_{2}\right)\right\}\right],{ }^{15}$ and previous attempts to use $\mathrm{BaI}_{2}$ as a metal source failed to result in any complexes. ${ }^{13}$ The reaction of $\left[\mathrm{Ba}(\mathrm{acacH})(\mathrm{MeCN})_{5}\right]\left[\mathrm{BAr}^{\mathrm{F}}\right]_{2}$ with [18] ane $\mathrm{O}_{2} \mathrm{~S}_{4}$ and [18] $\mathrm{aneO}_{4} \mathrm{Se}_{2}$ in $\mathrm{MeCN}$ produced the complexes $[\mathrm{Ba}($ heterocrown $)(\mathrm{acacH})(\mathrm{MeCN})]\left[\mathrm{BAr}^{\mathrm{F}}\right]_{2}$, in which the acacH ligand was retained, contrasting with the strontium complexes. This was confirmed by the crystal structure of $\left[\mathrm{Ba}\left([18] \mathrm{aneO}_{4} \mathrm{Se}_{2}\right)(\mathrm{acacH})(\mathrm{MeCN})\right]\left[\mathrm{BAr}^{\mathrm{F}}\right]_{2} \quad(\mathrm{Fig} . \quad 6)$ the first barium complex containing neutral selenoether coordination. The larger $\mathrm{Ba}^{2+}\left(1.43 \AA\right.$ ) compared to $1.27 \AA$ for $\mathrm{Sr}^{2+}$ (ref. 30) results in an increase in coordination number to nine, composed of the six donor atoms from the heterocrown, two oxygens from the neutral acacH and one $\mathrm{MeCN}$, in a geometry that can be described as distorted tricapped trigonal prismatic. Coordination numbers of 8-10 have been found in complexes of 18-crown- 6 with $\mathrm{Ba}^{2+}$ with similar bond lengths and irregular geometries. ${ }^{15,26,27,31-33}$

The complexes of these heterocrowns with the much smaller magnesium ion $\left(r^{2+}=0.78 \AA\right)$ were expected to differ from those described above. The reaction of $\left[\mathrm{Mg}(\mathrm{MeCN})_{6}\right]$ $\left[\mathrm{BAr}^{\mathrm{F}}\right]_{2}$ with 18-crown-6, [18] $\mathrm{aneO}_{2} \mathrm{~S}_{4}, \quad[18] \mathrm{aneO}_{4} \mathrm{~S}_{2}$ and [18] ane $\mathrm{O}_{4} \mathrm{Se}_{2}$ in anhydrous $\mathrm{CH}_{2} \mathrm{Cl}_{2}$ gave yellow or off-white solids of composition $\left[\mathrm{Mg}(\right.$ crown $\left.)(\mathrm{MeCN})_{2}\right]\left[\mathrm{BAr}^{\mathrm{F}}\right]_{2}$. Repeated attempts to obtain X-ray quality crystals were unsuccessful, although structures of two hydrolysis products were obtained (Fig. 6). The spectroscopic data on $\left[\mathrm{Mg}(\right.$ crown $\left.)(\mathrm{MeCN})_{2}\right]\left[\mathrm{BAr}^{\mathrm{F}}\right]_{2}$ (crown $=18$-crown-6, [18] $\mathrm{aneO}_{4} \mathrm{~S}_{2}$ and [18] $\mathrm{aneO}_{4} \mathrm{Se}_{2}$ ) show the presence of coordinated MeCN and the absence of water. The

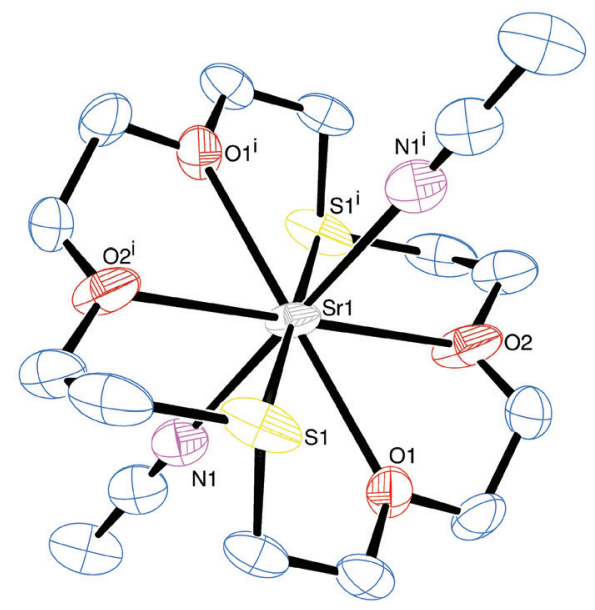

(a)

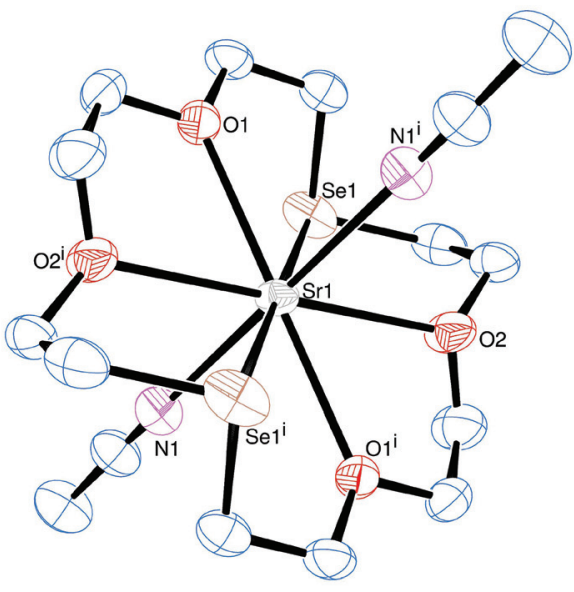

(b)

Fig. 5 (a) The cation of $\left[\mathrm{Sr}\left([18] a n e \mathrm{O}_{4} \mathrm{~S}_{2}\right)(\mathrm{MeCN})_{2}\right][\mathrm{BAr}]_{2}$ with the $[\mathrm{BAr}]^{-}$anions and hydrogen atoms omitted for clarity. Ellipsoids are shown at the $50 \%$ probability level. Selected bond lengths $(\AA)$ and angles $\left({ }^{\circ}\right)$ : $\mathrm{Sr}-\mathrm{O} 1=2.593(3), \mathrm{Sr}-\mathrm{O} 2=2.624(4), \mathrm{Sr}-\mathrm{S} 1=3.122(1), \mathrm{Sr}-\mathrm{N} 1=2.611(5) ; \mathrm{O} 1-\mathrm{Sr}-\mathrm{S} 1=$ 63.15(9), $\mathrm{O}^{i}-\mathrm{Sr}-\mathrm{S} 1=64.4(1), \mathrm{O} 1-\mathrm{Sr}-\mathrm{O} 2=61.8(1)$. Symmetry code: (i) $-x, 2-y, 2-z$. (b) The cation of $\left[\mathrm{Sr}\left([18] \mathrm{aneO}_{4} \mathrm{Se}_{2}\right)\left(\mathrm{MeCN}_{2}\right][\mathrm{BAr}]_{2}\right.$ with hydrogen atoms and $\left[\mathrm{BAr}^{\mathrm{F}}\right]^{-}$anions omitted for clarity. Ellipsoids are shown at the $50 \%$ probability level. Selected bond lengths $(\AA \AA)$ and angles $\left({ }^{\circ}\right)$ : $\mathrm{Sr}-$ $\mathrm{O} 1=2.606(3), \mathrm{Sr}-\mathrm{O} 2=2.611(3), \mathrm{Sr}-\mathrm{Se} 1=3.2216(6), \mathrm{Sr}-\mathrm{N} 1=2.620(4) ; \mathrm{O} 1-\mathrm{Sr}-\mathrm{Se} 1=65.02(7), \mathrm{O} 2-\mathrm{Sr}-\mathrm{Se} 1=65.59(7), \mathrm{O} 1-\mathrm{Sr}-\mathrm{O} 2^{i}=61.96(9) . \mathrm{Sym}-$ metry code: (i) $-x,-y,-z$. 


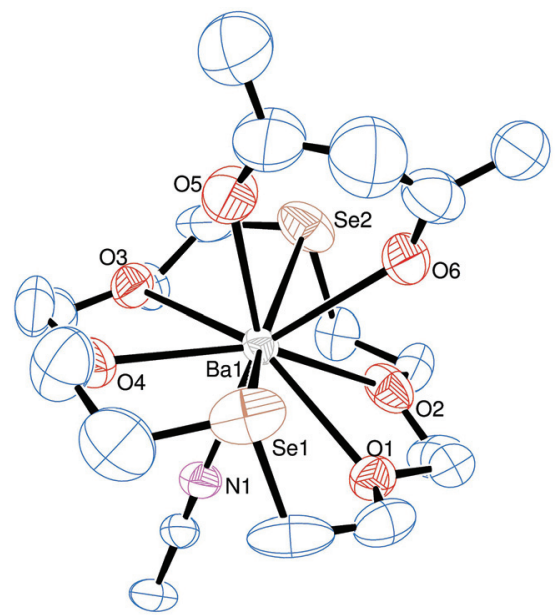

Fig. 6 The cation of $\left[\mathrm{Ba}\left([18] \mathrm{aneO}_{4} \mathrm{Se}_{2}\right)(\mathrm{acacH})(\mathrm{MeCN})\right]\left[\mathrm{BAr}^{\mathrm{F}}\right]_{2}$ with the positional disorder of the macrocycle, $\mathrm{CH}_{2} \mathrm{Cl}_{2}$ solvent molecule and hydrogen atoms omitted for clarity. Ellipsoids are shown at the 50\% probability level. Selected bond lengths $(\AA)$ and angles $\left({ }^{\circ}\right)$ : $\mathrm{Ba}-\mathrm{O} 1=$ 2.791(6), $\mathrm{Ba}-\mathrm{O} 2=2.786(7), \mathrm{Ba}-\mathrm{O} 3=2.787(6), \mathrm{Ba}-\mathrm{O} 4=2.813(7), \mathrm{Ba}-\mathrm{O} 5$ $=2.733(8), \mathrm{Ba}-\mathrm{O} 6=2.770(7), \mathrm{Ba}-\mathrm{Se} 1=3.363(1), \mathrm{Ba}-\mathrm{Se} 2=3.366(1), \mathrm{Ba}-$ $\mathrm{N} 1=2.920(8) ; \mathrm{Se} 1-\mathrm{Ba}-\mathrm{O} 1$ = 62.9(1), O1-Ba-O2 = 61.0(2), O2-Ba-Se2 $=60.8(2), \mathrm{Se} 2-\mathrm{Ba}-\mathrm{O} 3=62.3(2), \mathrm{O} 3-\mathrm{Ba}-\mathrm{O} 4=63.3(2), \mathrm{O} 4-\mathrm{Ba}-\mathrm{Se} 1=$ $63.8(2) \mathrm{O} 5-\mathrm{Ba} 1-\mathrm{O} 6=63.7(2)$.

${ }^{1} \mathrm{H}$ NMR resonances of the crown ligands show small high frequency shifts relative to the unbound ligands, ${ }^{18}$ but are rather broad. The $\mathrm{CH}_{2} \mathrm{O}$ and $\mathrm{CH}_{2} \mathrm{~S}$ resonances of $\left[\mathrm{Mg}\left([18] \mathrm{aneO}_{2} \mathrm{~S}_{4}\right)\right.$ $\left.(\mathrm{MeCN})_{2}\right]\left[\mathrm{BAr}^{\mathrm{F}}\right]_{2}$ are very broad both in the ${ }^{1} \mathrm{H}$ NMR and ${ }^{13} \mathrm{C}\left\{{ }^{1} \mathrm{H}\right\}$ spectra which probably indicates substantial solution lability of the heterocrown, which contains four soft thioether donors on the hard magnesium centre. Since the steric demands of the linear MeCN groups are small, it is possible that the $\left[\mathrm{Mg}(\text { crown })(\mathrm{MeCN})_{2}\right]^{2+}$ cations contain eight-coordinate magnesium in the solid state, although the coordination number could be lower, whilst the ring undergoes a fast dynamic process in solution. Seven-coordination is known with $\kappa^{5}$-18-crown-6 in $\left[\mathrm{Mg}\left(18 \text {-crown-6) }\left(\mathrm{HCl}_{2}\right)_{2}\right]^{34}\right.$ and $\left[\mathrm{Mg}\left(\kappa^{5}-18\right.\right.$-crown-6) $\left.\mathrm{Cl}_{2}\right],{ }^{35}$ which contain near planar $\mathrm{MgO}_{5}$ units with trans coordinated anions above and below the plane.

During attempts to grow crystals of $\left[\mathrm{Mg}(\right.$ crown $\left.)(\mathrm{MeCN})_{2}\right]\left[\mathrm{BAr}^{\mathrm{F}}\right]_{2}$ from $\mathrm{CH}_{2} \mathrm{Cl}_{2} / n$-hexane in a freezer, crystals of $[\mathrm{Mg}$ (crown)$\left.(\mathrm{MeCN})\left(\mathrm{OH}_{2}\right)_{2}\right]\left[\mathrm{BAr}^{\mathrm{F}}\right]_{2}\left(\right.$ crown $=[18] \mathrm{aneO}_{4} \mathrm{~S}_{2}$ or $\left.[18] \mathrm{aneO}_{4} \mathrm{Se}_{2}\right)$ were obtained, no doubt due to ingress of adventitious water. The structures are isomorphous (Fig. 7) and contain $\kappa^{3}$-crown, one MeCN and two water ligands. The crown is coordinated $\mathrm{O}_{2} \mathrm{~S} / \mathrm{Se}$ in a mer geometry - providing extremely rare examples of $\mathrm{Mg}^{2+}$ coordinated to neutral thio- and seleno-ether functions, with cis-diaquo ligands. Hydrogen-bonding is present between the coordinated $\mathrm{H}_{2} \mathrm{O}$ ligands and the remaining O-atoms of the crown. $\kappa^{3}$-Coordination of 18 -crown- 6 is present in the five-coordinate $\left[\mathrm{Mg}\left(18\right.\right.$-crown-6) $\left({ }^{\mathrm{i}} \mathrm{Bu}\right)$ $\left.\left(2,6{ }^{t}{ }^{t} \mathrm{Bu}_{2} \mathrm{C}_{6} \mathrm{H}_{4} \mathrm{O}\right)\right] .{ }^{36}$

Adventitious hydrolysis of some $[\mathrm{M}$ (heterocrown $\left.) \mathrm{I}_{2}\right](\mathrm{M}=$ $\mathrm{Ca}$ or $\mathrm{Sr}$ ) complexes displaced the iodide ligands to give $[\mathrm{M}$ (heterocrown $\left.)\left(\mathrm{OH}_{2}\right)_{2}\right] \mathrm{I}_{2}{ }^{13}$ but $\kappa^{6}$-coordination of the crown remained. The different behaviour of $\mathrm{Mg}$ versus $\mathrm{Ca}$ and $\mathrm{Sr}$ in these systems may be largely attributable to the smaller size of the magnesium. We note that mixed aqua-crown complexes are also well established for some $3 \mathrm{~d}$ metals, e.g. $\left[\mathrm{Cr}\left(\kappa^{2}-18\right.\right.$-crown-6) $\left.\left(\mathrm{OH}_{2}\right) \mathrm{Cl}_{3}\right]$ or $\left[\mathrm{V}\left(\kappa^{2}-18\right.\right.$-crown-6) $\left.\left(\mathrm{OH}_{2}\right) \mathrm{Cl}_{3}\right]$. $^{37,38}$

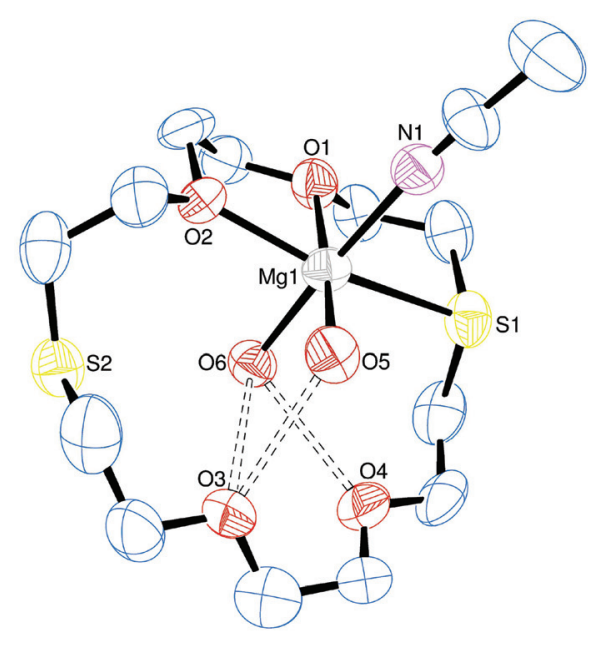

(a)

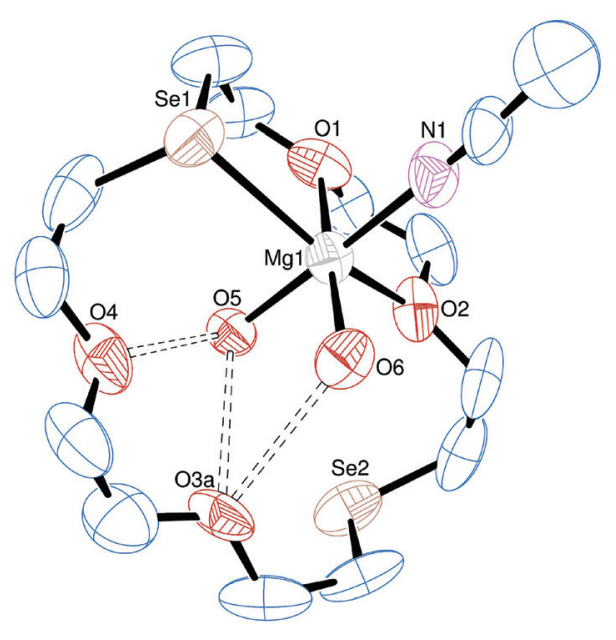

(b)

Fig. 7 (a) The cation of $\left[\mathrm{Mg}\left(\mathrm{K}^{3}-[18] \mathrm{aneO} \mathrm{S}_{2}\right)\left(\mathrm{OH}_{2}\right)_{2}(\mathrm{MeCN})\right][\mathrm{BAr}]_{2}$, with positional disorder of the macrocycle and all hydrogen atoms omitted for clarity. Selected bond lengths $(\AA \AA)$ and angles $\left({ }^{\circ}\right): \mathrm{Mg}-\mathrm{O} 1=2.113(8), \mathrm{Mg}-\mathrm{O} 2=2.081(7), \mathrm{Mg}-\mathrm{S} 1=2.625(4), \mathrm{Mg}-\mathrm{O} 5=2.025(8), \mathrm{Mg}-\mathrm{O} 6=2.037(8)$, $\mathrm{Mg}-\mathrm{N} 1=2.14(1) ; \mathrm{S} 1-\mathrm{Mg}-\mathrm{O} 2=158.5(3), \mathrm{O} 1-\mathrm{Mg}-\mathrm{O} 2=77.5(3), \mathrm{S} 1-\mathrm{Mg}-\mathrm{O} 1=81.2(2)$. (b) The cation of $\left.\left[\mathrm{Mg}^{3} \mathrm{~K}^{3}-[18] \mathrm{aneO}_{4} \mathrm{Se}_{2}\right)\left(\mathrm{OH}_{2}\right)_{2}\left(\mathrm{MeCN}^{2}\right)\right][\mathrm{BAr}]_{2}$ with the $\left[\mathrm{BAr}^{\mathrm{F}}\right]^{-}$anions, positional disorder of the macrocycle and hydrogens omitted for clarity. Selected bond lengths $(\AA \AA \AA)$ and angles $\left({ }^{\circ}\right): \mathrm{Mg}-\mathrm{O} 1=$ 2.107(8), $\mathrm{Mg}-\mathrm{O} 2=2.064(8), \mathrm{Mg}-\mathrm{Se} 1=2.794(4), \mathrm{Mg}-\mathrm{O} 5=2.027(8), \mathrm{Mg}-\mathrm{O} 6=2.022(8), \mathrm{Mg}-\mathrm{N} 1=2.12(1) ; \mathrm{Se} 1-\mathrm{Mg}-\mathrm{O} 2=158.6(3), \mathrm{O} 1-\mathrm{Mg}-\mathrm{O} 2=$ 77.9(4), Se1-Mg-O1 = 80.8(3). 


\section{Conclusions}

A range of cationic complexes of the Group 2 metals $\mathrm{Mg}-\mathrm{Ba}$ with heterocrown ethers have been prepared from acetonitrile synthons taking advantage of the solubility in weak donor solvents conferred by the $\left[\mathrm{BAr}^{\mathrm{F}}\right]^{-}$anions. The new complexes have been fully characterised spectroscopically and in many cases by X-ray crystallography. They include the first example of coordination of a neutral selenoether function to $\mathrm{Ba}^{2+}$, and rare examples with seleno- and thio-ether coordination to the other hard metal centres. The heterocrown-nitrile complexes should prove to be useful synthons for incorporation of other donor types to the metal centre, as demonstrated by the characterisation of $\left[\mathrm{Ca}\left([18] \mathrm{aneO}_{4} \mathrm{~S}_{2}\right)(\right.$ bipy $\left.)\right]\left[\mathrm{BAr}^{\mathrm{F}}\right]_{2}$, whilst more generally, the $\left[\mathrm{M}(\mathrm{MeCN})_{x}\right]\left[\mathrm{BAr}^{\mathrm{F}}\right]_{2}(\mathrm{M}=\mathrm{Mg}, x=6 ; \mathrm{M}=\mathrm{Ca}, x=8)$ or $\left[\mathrm{M}^{\prime}(\mathrm{acacH})(\mathrm{MeCN})_{5}\right]\left[\mathrm{BAr}^{\mathrm{F}}\right]_{2}\left(\mathrm{M}^{\prime}=\mathrm{Sr}, \mathrm{Ba}\right)$ may offer a route into other Group 2 complexes with soft donor atoms. Further work to explore these possibilities is currently underway.

\section{Acknowledgements}

We thank the EPSRC for support via the SCFED project through a Programme Grant (EP/1033394/1), and also through EP/K039466/1. The SCFED Project (http://www.scfed.net) is a multidisciplinary collaboration of British universities investigating the fundamental and applied aspects of supercritical fluids. Additional Data Available: Original IR and NMR spectra for all complexes are available to download via http://dx.doi.org/ 10.5258/SOTON/391234.

\section{References}

1 N. N. Greenwood and A. Earnshaw, Chemistry of the Elements, Butterworth, Oxford, 2nd edn, 1997; F. A. Cotton, G. Wilkinson, C. A. Murillo and M. Bochmann, Advanced Inorganic Chemistry, Wiley, NY, 1999.

2 D. E. Fenton, in Comprehensive Coordination Chemistry, ed. G. Wilkinson, R. D. Gillard and J. A. McCleverty, Pergamon Press, Oxford, 1987, vol. 3, p. 25.

3 W. E. Lindsell, in Comprehensive Organometallic Chemistry II, ed. G. Wilkinson, F. G. A. Stone and E. W. Abel, Elsevier, Oxford, 1995, vol. 1, p. 57.

4 T. P. Hanusa, Coord. Chem. Rev., 2000, 31, 143; T. P. Hanusa, in Comprehensive Coordination Chemistry II, ed. J. A. McCleverty and T. J. Meyer, Elsevier, Oxford, 2004, vol. 3, p. 1; A. Torvisco, A. Y. O'Brien and K. Ruhlandt-Senge, Coord. Chem. Rev., 2011, 255, 1268; M. Westerhausen, S. Krieck, J. Langer, T. M. A. Al-Shboul and H. Gorls, Coord. Chem. Rev., 2013, 257, 1049; S. Harder, Chem. Rev., 2010, 110, 3852.

5 T. P. Hanusa, in Comprehensive Organometallic Chemistry III, ed. R. H. Crabtree and D. M. P. Mingos, Elsevier, Oxford, 2007, vol. 2, p. 68; A. Torvisco and K. RuhlandtSenge, Top. Organomet. Chem., 2013, 45, 1.
6 Handbook of Grignard Reagents, ed. G. S. Silverman and P. E. Rakita, Marcel Dekker, New York, 1996; H. G. Richey, Grignard Reagents: New Developments, Wiley, New York, 2000.

7 A. F. Waters and A. H. White, Aust. J. Chem., 1996, 49, 27.

8 M. Carravetta, M. Concistre, W. Levason, G. Reid and W. Zhang, Chem. Commun., 2015, 51, 9555.

9 M. J. D. Champion, J. M. Dyke, W. Levason, M. E. Light, D. Pugh, H. Bhakhoa, L. Rhyman, P. Ramasami and G. Reid, Inorg. Chem., 2015, 54, 2497.

10 M. Everett, A. Jolleys, W. Levason, D. Pugh and G. Reid, Chem. Commun., 2014, 50, 5843; J. M. Dyke, W. Levason, M. E. Light, D. Pugh, G. Reid, H. Bhakhoa, P. Ramasami and L. Rhyman, Dalton Trans., 2015, 44, 13853.

11 M. J. D. Champion, W. Levason, D. Pugh and G. Reid, Dalton Trans., 2015, 43, 18748.

12 W. Levason and G. Reid, in Supramolecular Chemistry: From molecules to nanomaterials, ed. P. A. Gale and J. W. Steed, Wiley, New York, 2012, vol. 3, p. 785.

13 P. Farina, W. Levason and G. Reid, Dalton Trans., 2013, 42, 89 .

14 I.-H. Park, K.-M. Park and S. S. Lee, Dalton Trans., 2010, 39, 9696.

15 T. Roettgers and W. S. Sheldrick, Z. Anorg. Allg. Chem., 2001, 627, 1976.

16 M. V. Alfimov, A. V. Churakov, Y. V. Fedorov, O. A. Fedorova, S. P. Gromov, J. A. K. Howard, L. G. Kuz'mina, D. Mobius, T. I. Sergeevad, A. I. Vedernikov, O. V. Yescheulova and S. Y. Zaitsev, New J. Chem., 2002, 26, 543.

17 M. Brookhart, B. Grant and A. F. Volpe Jr., Organometallics, 1992, 11, 30866.

18 P. Farina, T. Latter, W. Levason and G. Reid, Dalton Trans., 2013, 42, 4714.

19 CrystalClear-SM Expert 3.1 b27, Rigaku Corporation, Tokyo, Japan, 2012; CrystalClear-SM Expert 2.1 b29, Rigaku Corporation, Tokyo, Japan, 2013.

20 L. J. Farrugia, J. Appl. Crystallogr., 2012, 45, 849.

21 F. H. Allen, O. Johnson, G. P. Shields, B. R. Smith and M. Towler, J. Appl. Crystallogr., 2004, 37, 335.

22 Y. Nakamura, K. Isobe, H. Morita, S. Yamazaki and S. Kawaguchi, Inorg. Chem., 1972, 7, 1573.

23 B. A. Stork-Blaisse, G. C. Verschoor and C. Romers, Acta Crystallogr., Sect. B: Struct. Crystallogr. Cryst. Chem., 1972, 8, 2445.

24 B. Swanson and D. F. Shriver, Inorg. Chem., 1970, 9, 1406.

25 Elemental analyses show that $\left[\mathrm{M}(\mathrm{MeCN})_{5} \mathrm{I}_{2}\right](\mathrm{M}=\mathrm{Ca}, \mathrm{Sr})$ also lose some nitrile ligands on drying - see ref. 7.

26 A. Verma, M. Guino-o, M. Gillett-Kunnath, W. Teng and K. Ruhlandt-Senge, Z. Anorg. Allg. Chem., 2009, 635, 903.

27 J. Langer, S. Krieck, R. Fischer, H. Goerls and M. Westerhausen, Z. Anorg. Allg. Chem., 2010, 636, 1190.

28 D. L. Kepert, Prog. Inorg. Chem., 1978, 24, 179.

29 S. J. Lippard and B. J. Russ, Inorg. Chem., 1968, 7, 1686.

30 J. Emsley, The Elements, OUP, Oxford, 1989.

31 P. C. Junk and J. W. Steed, J. Coord. Chem., 2007, 60, 1017.

32 M.-M. Zhao, Acta Crystallogr., Sect. E: Struct. Rep. Online, 2012, 68, m286. 
33 M. D. Brown, M. F. Davis, J. M. Dyke, F. Ferranti, W. Levason, J. S. Ogden and M. Webster, Chem. - Eur. J., 2008, 14, 2615.

34 J. L. Atwood, S. G. Bott, C. M. Means, A. W. Coleman, H. Zhang and J. T. May, Inorg. Chem., 1999, 29, 467.

35 N. R. Streltsova, L. V. Ivakina, P. A. Storozhenko, B. M. Bulychev and V. K. Bel'ski, Dokl. Akad. Nauk SSSR, 1986, 291, 1373.
36 A. D. Parjerski, E. P. Squiller, M. Parvez, R. D. Whittle and H. G. Richey Jr., Organometallics, 2005, 24, 809.

37 U. Kynast, S. G. Bott and J. L. Atwood, J. Coord. Chem., 1988, 17, 53.

38 C. D. Beard, L. Carr, M. F. Davis, J. Evans, W. Levason, L. D. Norman, G. Reid and M. Webster, Eur. J. Inorg. Chem., 2006, 4399. 